\title{
ANALYTIC BUNDLE STRUCTURE ON THE IDEMPOTENT MANIFOLD
}

\author{
CHI-WAI LEUNG AND CHI-KEUNG NG
}

\begin{abstract}
Let $X$ be a (real or complex) Banach space, and $\mathcal{J}(X)$ be the set of all (non-zero and non-identity) idempotents; i.e., bounded linear operators on $X$ whose squares equal themselves. We show that the Banach submanifold $\mathcal{J}(X)$ of $\mathcal{L}(X)$ is a locally trivial analytic affine-Banach bundle over the Grassmann manifold $\mathscr{G}(X)$, via the map $\kappa$ that sends $Q \in \mathcal{J}(X)$ to $Q(X)$, such that the affine-Banach space structure on each fiber is the one induced from $\mathcal{L}(X)$ (in particular, every fiber is an affine-Banach subspace of $\mathcal{L}(X))$.

Using this, we show that if $K$ is a real Hilbert space, then the assignment

$$
(E, T) \mapsto T^{*} \circ P_{E^{\perp}}+P_{E}, \quad \text { where } E \in \mathscr{G}(K) \text { and } T \in \mathcal{L}\left(E, E^{\perp}\right)
$$

induces a bi-analytic bijection from the total space of the tangent bundle, $\mathbf{T}(\mathscr{G}(K))$, of $\mathscr{G}(K)$ onto $\mathcal{J}(K)$ (here, $E^{\perp}$ is the orthogonal complement of $E, P_{E} \in \mathcal{L}(K)$ is the orthogonal projection onto $E$, and $T^{*}$ is the adjoint of $T$ ). Notice that this bi-analytic bijection is an affine map on each tangent plane.
\end{abstract}

\section{INTRODUCTION}

The Grassmannian of a finite dimensional vector space is a very well-studied object. This manifold is important in both pure and applied mathematics (see e.g. $[5,6,7,12,17,18,19,20,22,25,28$, $29,33]$ for some recent accounts on it). There are two main streams of generalizations of the Grassmannian to the infinite dimensional case. The first one was introduced by Douady in [15] (see also [23] and [32]). In this case, the Grassmannian of a (either real or complex) Banach space $X$ is the set of complemented subspaces of $X$ equipped with a canonical (respectively, real or complex) analytic Banach manifold structure such that a local chart around a subspace is given by the Banach space of continuous linear operators from that subspace to a complement of it. Another approach was first appeared in the work of Porta and Recht in [30]. In this approach, the Grassmannian of a Banach algebra $D$ is defined to be the set of equivalence classes of idempotents under certain equivalence relation, and is equipped with the quotient topology. In the particular case when $D$ is a $C^{*}$-algebra, the Grassmannian can be identified, as a topological space, with the set of (self-adjoint) projections, and the later is a real analytic Banach submanifold of $D$ (see e.g., [2], [3] and [8]; see also [9], [11] and [24] for the generalizations to $J B$-algebras and $J B^{*}$-triples). A connection between the two approaches was obtain [1], where it was shown that there is a real bi-analytic bijection from the Grassmannian of a complex Hilbert space $H$ (in the sense of Douady) to the set of self-adjoint projections of the $C^{*}$-algebra $\mathcal{L}(H)$ of continuous linear operators on $H$.

We will follows [15] for the definition of the Grassmann manifold $\mathscr{G}(X)$ of a (either real or complex) Banach space $X$. We denote by $\mathcal{J}(X)$ the set of all non-zero proper idempotents in the Banach manifold $\mathcal{L}(X)$ of all bounded linear operators. Notice that "idempotents" were also called "projections" in some literature, but we prefer the term "idempotents" in order to distinguish them with "self-adjoint projections" in the case of Hilbert spaces (which will also be considered in this article). We define $\kappa: \mathcal{J}(X) \rightarrow \mathscr{G}(X)$ as follows:

$$
\kappa(Q):=Q(X) \quad(Q \in \mathcal{J}(X))
$$

Date: January 9, 2020.

2010 Mathematics Subject Classification. Primary: 46T05, 57N20, 58D15.

Key words and phrases. Infinite dimensional Grassmannian, Idempotents, Banach bundles, Affine-Banach spaces, Tangent bundles.

THIS WORK IS SUPPORTED BY THE NATIONAL NATURAL SCIENCE FOUNDATION OF CHINA (11471168) AND (11871285). 
The main results of this paper can be summarized in the following (see Theorem 3.4, Proposition 4.1 and Proposition 4.3).

Theorem 1. Let $X$ be a real or complex Banach space, which is not one-dimensional.

(a) Under the Banach submanifold structure on $\mathcal{J}(X)$ induced from $\mathcal{L}(X)$, one knows that $\kappa: \mathcal{J}(X) \rightarrow$ $\mathscr{G}(X)$ is a locally trivial (respectively, real or complex) analytic affine-Banach bundle, such that the affine-Banach space structures on the fibers of $\kappa$ are the ones induced from $\mathcal{L}(X)$.

(b) There exist equivalent Banach space structures on the fiber of $\kappa$, under which $\kappa: \mathcal{J}(X) \rightarrow \mathscr{G}(X)$ becomes a locally trivial continuous Banach bundle.

(c) In the case when $X$ is a complex Banach space, there can never exist Banach space structures on the fiber of $\kappa$ such that $\kappa: \mathcal{J}(X) \rightarrow \mathscr{G}(X)$ becomes a locally trivial complex analytic Banach bundle.

In the case of a real Hilbert space $K$, we also obtain the following:

there is an analytic immersion from the total space of the tangent bundle of $\mathscr{G}(K)$

to $\mathcal{L}(K)$ such that the restriction of this immersion on each fiber is affine.

More precisely, elements in the tangent bundle of $\mathscr{G}(K)$ can be identified with a pair $(E, T)$, where $E \in \mathscr{G}(K)$ and $T$ is a bounded linear operator from $E$ to the orthogonal complement $E^{\perp}$ of $E$. The following is obtained in Theorem 4.5 and Corollary 4.6(a).

Theorem 2. Let $K$ be a real Hilbert space, and $\mathbf{T}(\mathscr{G}(K))$ be the tangent bundle of $\mathscr{G}(K)$.

(a) The assignment $Q \mapsto\left(Q(X),\left.\bar{P}_{Q(X) \perp} \circ Q^{*}\right|_{Q(X)}\right)$, where $\bar{P}_{Q(X) \perp}: K \rightarrow Q(X)^{\perp}$ is the orthogonal projection, is a bi-analytic bijection from $\mathrm{J}(K)$ onto $\mathbf{T}(\mathscr{G}(K))$ such that for each $E \in \mathscr{G}(K)$, this bijection is an affine map from $\{Q \in \mathcal{J}(K): Q(K)=E\}$ onto the tangent plane over $E$.

(b) The assignment $(E, T) \mapsto\left(E, T \circ \bar{P}_{E}\right)$ is a fiberwise linear bi-analytic map from $\mathbf{T}(\mathscr{G}(K))$ onto the Banach subbundle $\left\{(E, S): E \in \mathscr{G}(K) ; S \in \mathcal{L}^{E^{\perp}}\left(K, E^{\perp}\right)\right\}$ of the trivial Banach bundle $(\mathscr{G}(K) \times$ $\left.\mathcal{L}(K), \mathscr{G}(K), \kappa_{0}\right)$; here $\mathcal{L}^{F}(K, F):=\{S \in \mathcal{L}(K): T(F)=\{0\} ; T(K) \subseteq F\}$.

On our way, we also obtain that $\mathcal{J}(X)$ (under the norm topology) is canonically homeomorphic to the following subspace of the product topological space $\mathscr{G}(X) \times \mathscr{G}(X)$ (see Corollary 3.6):

$$
\{(E, F) \in \mathscr{G}(X) \times \mathscr{G}(X): E \text { and } F \text { are complements to each other }\}
$$

Furthermore, similar to the corresponding fact for $\mathscr{G}(X)$, we will show, in Corollary 5.3 , that each orbit in $\mathcal{J}(X)$ under the canonical action by the Banach Lie group $G \mathcal{L}(X)$ of continuous invertible operators on $X$ is a clopen subset, and can be identified bi-analytically with a homogeneous space of $G \mathcal{L}(X)$. We will also verify in Proposition 5.4 that, for any $n \in \mathbb{N}$, the set $\{Q \in \mathcal{J}(X): \operatorname{dim} Q(X)=$ $n\}$ is a connected component of $\mathcal{J}(X)$.

Using the above, for $n \in \mathbb{N}$ and $k \in\{1, \ldots, n\}$, if the map

$$
v: \mathrm{GL}_{n}(\mathbb{R}) / \mathrm{GL}_{k}(\mathbb{R}) \times \mathrm{GL}_{n-k}(\mathbb{R}) \rightarrow \mathrm{O}_{n} / \mathrm{O}_{k} \times \mathrm{O}_{n-k}
$$

is given by the Gram-Schmidt process (on column vectors), then $v$ induces a locally trivial real analytic vector bundle structure on the homogeneous space $\mathrm{GL}_{n}(\mathbb{R}) / \mathrm{GL}_{k}(\mathbb{R}) \times \mathrm{GL}_{n-k}(\mathbb{R})$, which can be identified with the tangent bundle of $\mathrm{O}_{n} / \mathrm{O}_{k} \times \mathrm{O}_{n-k}$ (see Example 5.5(b)).

\section{Notations and Preliminary}

Let us begin this paper by giving some notation. Throughout this article, $\mathbb{K}$ is either the real field $\mathbb{R}$ or the complex field $\mathbb{C}$. If $X$ and $Y$ are $\mathbb{K}$-Banach spaces, we denote by $\mathcal{L}(X, Y)$ the Banach space of all continuous $\mathbb{K}$-linear operators from $X$ to $Y$. We will also denote $\mathcal{L}(X):=\mathcal{L}(X, X)$. Moreover, the identity map in $\mathcal{L}(X)$ will be denoted by $I_{X}$, and sometimes by $I$ if no confusion arise.

Unless specified otherwise, by $\mathbb{K}$-Banach manifolds, we mean $\mathbb{K}$-analytic Banach manifolds, in the sense of [32] and [10]. 
Throughout this article, $\mathscr{G}(X)$ is the collection of all non-zero proper complemented subspaces of $X$. For any $E \in \mathscr{G}(X)$, we denote $F \top E$ if $F$ is a complement of $E$, and put

$$
\mathcal{C}_{E}:=\{F \in \mathscr{G}(X): F \top E\} .
$$

We set, as in [32, p.44-46],

$$
p_{E, F}=q_{E, F}^{-1},
$$

where $q_{E, F}: \mathcal{L}(E, F) \rightarrow \mathcal{C}_{F}$ is the bijection given by

$$
q_{E, F}(T):=(I+T)(E) \quad(T \in \mathcal{L}(E, F)) .
$$

There is a Hausdorff metrizable topology on $\mathscr{G}(X)$ such that $\mathcal{C}_{F}$ is an open subset of $\mathscr{G}(X)$ and $p_{E, F}: \mathcal{C}_{F} \rightarrow \mathcal{L}(E, F)$ is a homeomorphism. Moreover,

$$
\left\{\left(\mathcal{C}_{F_{0}}, p_{E_{0}, F_{0}}, \mathcal{L}\left(E_{0}, F_{0}\right)\right): E_{0}, F_{0} \in \mathscr{G}(X) ; F_{0} \top E_{0}\right\}
$$

constitutes an analytic atlas for a $\mathbb{K}$-Banach manifold structure on $\mathscr{G}(X)$. When equipped with this structure, $\mathscr{G}(X)$ is called the Grassmann manifold of $X$.

A subset $A \subseteq X$ is called a $\mathbb{K}$-affine-Banach subspace if $A-a_{0}$ is a $\mathbb{K}$-Banach subspace of $X$ for one (equivalently, for every) element $a_{0} \in A$. Moreover, a map $S$ from $A$ to a $\mathbb{K}$-Banach space $Y$ is said to be $\mathbb{K}$-affine if the assignment

$$
S^{a_{0}}: a-a_{0} \mapsto S(a)-S\left(a_{0}\right)
$$

is a $\mathbb{K}$-linear map on $A-a_{0}$. We say that $A$ is isometrically affine isomorphic to $Y$ if there is a $\mathbb{K}$-affine bijection $T: A \rightarrow Y$ which preserves the metrics, i.e., $\|T(a)-T(b)\|_{Y}=\|a-b\|_{X}(a, b \in A)$.

We denote by $\mathcal{A}(A, B)$ the set of all continuous $\mathbb{K}$-affine maps from $A$ to a $\mathbb{K}$-affine-Banach subspace $B$ of $Y$. It is clear that $\mathcal{A}(A, B)$ is a $\mathbb{K}$-vector space. The function $\|\cdot\|_{a_{0}}$ defined by

$$
\|T\|_{a_{0}}:=\left\|T^{a_{0}}\right\|+\left\|T\left(a_{0}\right)\right\| \quad(T \in \mathcal{A}(A, B))
$$

is a complete norm on $\mathcal{A}(A, B)$.

The following well-known fact ensures a default Banach space structure on $\mathcal{A}(A, B)$ up to Banach space isomorphism.

Lemma 2.1. For any $a_{0}, a_{1} \in A$, the two norms $\|\cdot\|_{a_{0}}$ and $\|\cdot\|_{a_{1}}$ are equivalent.

This paper mainly concerns with affine-Banach bundles. We need to consider such a general notion (instead of the more well-known notion of Banach bundles) because the set of idempotents naturally forms an affine-Banach bundle. Since affine-Banach bundles are not well-documented, let us give its precise definition below.

Definition 2.2. Let $\Omega$ and $\Upsilon$ be Hausdorff spaces. Let $\kappa: \Upsilon \rightarrow \Omega$ be a continuous surjection.

(a) Then $(\Upsilon, \Omega, \kappa)$ is called a locally trivial continuous $\mathbb{K}$-affine-Banach bundle (respectively, locally trivial continuous $\mathbb{K}$-Banach bundle) if the following conditions are satisfied:

B1) for each $\omega \in \Omega$, the subset

$$
\Upsilon_{\omega}:=\kappa^{-1}(\omega)
$$

is homeomorphic to a $\mathbb{K}$-affine-Banach subspace of a $\mathbb{K}$-Banach space (respectively, homeomorphic to a $\mathbb{K}$-Banach space);

B2) for each $\omega_{0} \in \Omega$, there exist an open neighborhood $V_{\omega_{0}} \subseteq \Omega$ of $\omega_{0}$ as well as a bi-continuous bijection $\Theta_{\omega_{0}}: V_{\omega_{0}} \times \Upsilon_{\omega_{0}} \rightarrow \kappa^{-1}\left(V_{\omega_{0}}\right)$ such that $\Theta_{\omega_{0}} \mid\{\omega\} \times \Upsilon_{\omega_{0}}$ is a $\mathbb{K}$-affine map (respectively, $\mathbb{K}$-linear map) onto $\Upsilon_{\omega}$, for every $\omega \in V_{\omega_{0}}$;

B3) for $\omega_{1}, \omega_{2} \in \Omega$ with $V_{\omega_{1}} \cap V_{\omega_{2}} \neq \emptyset$, the map $\varphi: V_{\omega_{1}} \cap V_{\omega_{2}} \rightarrow \mathcal{A}\left(\Upsilon_{\omega_{1}}, \Upsilon_{\omega_{2}}\right)$ defined by

$$
\varphi(\omega)(x):=\Pi_{2}\left(\Theta_{\omega_{2}}^{-1} \circ \Theta_{\omega_{1}}(\omega, x)\right) \quad\left(\omega \in V_{\omega_{1}} \cap V_{\omega_{2}} ; x \in \Upsilon_{\omega_{1}}\right)
$$

is continuous, where $\Pi_{2}$ is the projection onto the second coordinate. 
(b) Suppose that $\Omega$ and $\Upsilon$ are $\mathbb{K}$-Banach manifolds. Then $(\Upsilon, \Omega, \kappa)$ is called a locally trivial $\mathbb{K}$ analytic affine-Banach bundle (respectively, locally trivial $\mathbb{K}$-analytic Banach bundle), if $\kappa$ is $\mathbb{K}$ analytic and the same requirements as in part (a) hold with the terms "bi-continuous" and "continuous" in (B2) and (B3) being replaced by "KK-bi-analytic" and "KK-analytic", respectively.

In the case of a Banach bundle, Condition (B3) is equivalent to the corresponding statement when $\mathcal{A}\left(\Upsilon_{\omega_{1}}, \Upsilon_{\omega_{2}}\right)$ is replaced by $\mathcal{L}\left(\Upsilon_{\omega_{1}}, \Upsilon_{\omega_{2}}\right)$ (because of Lemma 2.1).

We may occasionally use the term "locally trivial continuous affine-Banach bundle" and "locally trivial analytic affine-Banach bundle" etc, if the underlying field $\mathbb{K}$ is understood.

A map $\rho$ from an open subset $V_{0} \subseteq \Omega$ to $\Upsilon$ is called a local cross section if

$$
\kappa(\rho(\omega))=\omega \quad\left(\omega \in V_{0}\right) .
$$

In the case when $V_{0}=\Omega$, we say that $\rho$ is a global cross section.

For a continuous (respectively, analytic) Banach bundle, the constant zero map is obviously a continuous (respectively, analytic) global cross section. The following proposition tells us that the only obstruction for an affine-Banach bundle to be a Banach bundle is the existence of global continuous (respectively, analytic) cross sections.

Proposition 2.3. (a) Let $(\Upsilon, \Omega, \kappa)$ be a locally trivial continuous affine-Banach bundle. If there is a continuous global cross section $\rho: \Omega \rightarrow \Upsilon$, then there exist Banach space structures on all the fibers of $\kappa$, which are isometrically affine isomorphic to the original affine-Banach space structures on the fibers, such that $(\Upsilon, \Omega, \kappa)$ becomes a locally trivial continuous Banach bundle.

(b) Let $(\Upsilon, \Omega, \kappa)$ be a locally trivial analytic affine-Banach bundle. If there is an analytic global cross section $\rho: \Omega \rightarrow \Upsilon$, then $(\Upsilon, \Omega, \kappa)$ is as a locally trivial analytic Banach bundle, under a Banach space structure on each fiber of $\kappa$ that is isometrically affine isomorphic to the original affine-Banach space structure on the fiber.

Although this proposition could be a known fact (at least in the finite dimensional case), we nevertheless give a brief account of it here. Fix $\omega_{0} \in \Omega$. The affine-Banach space $\Upsilon_{\omega_{0}}$ becomes a Banach space when equipped with the following structure:

$$
\|x\|_{\omega_{0}}:=\left\|x-\rho\left(\omega_{0}\right)\right\|, \quad \alpha \odot_{\omega_{0}} x:=\alpha x+(1-\alpha) \rho\left(\omega_{0}\right) \quad \text { and } \quad x \oplus_{\omega_{0}} y:=x+y-\rho\left(\omega_{0}\right),
$$

where $x, y \in \Upsilon_{\omega_{0}}, \alpha \in \mathbb{K}$ and $\|\cdot\|$ is the norm on the Banach space containing $\Upsilon_{\omega_{0}}$. Let $V_{\omega_{0}}$, $\Theta_{\omega_{0}}$ and $\Pi_{2}$ be as in Definition 2.2. Set $\zeta_{\omega_{0}}: V_{\omega_{0}} \rightarrow \Upsilon_{\omega_{0}}$ to be the map $\left.\Pi_{2} \circ \Theta_{\omega_{0}}^{-1} \circ \rho\right|_{\omega_{\omega_{0}}}$. By the continuity (respectively, analyticity) assumption on $\Theta_{\omega_{0}}^{-1}$ and $\rho$, the map $\zeta_{\omega_{0}}$ is continuous (respectively, analytic). Define $\Psi_{\omega_{0}}: V_{\omega_{0}} \times \Upsilon_{\omega_{0}} \rightarrow \kappa^{-1}\left(V_{\omega_{0}}\right)$ by

$$
\Psi_{\omega_{0}}(\omega, y):=\Theta_{\omega_{0}}\left(\omega, y \oplus_{\omega_{0}} \zeta_{\omega_{0}}(\omega)\right) \quad\left(\omega \in V_{\omega_{0}} ; y \in \Upsilon_{\omega_{0}}\right) .
$$

Clearly, $\Psi_{\omega_{0}}$ is continuous (respectively, analytic). It is not hard to verify that $\Psi_{\omega_{0}}$ is fiberwise linear. Furthermore, one can check that

$$
\Psi_{\omega_{0}}^{-1}(x)=\Theta_{\omega_{0}}^{-1}(x) \boxplus\left(\kappa(x), 2 \rho\left(\omega_{0}\right)-\zeta_{\omega_{0}}(\kappa(x))\right) \quad\left(x \in \kappa^{-1}\left(V_{\omega_{0}}\right)\right),
$$

where $(\omega, a) \boxplus(\omega, b):=\left(\omega, a \oplus_{\omega_{0}} b\right)\left(\omega \in V_{\omega_{0}} ; a, b \in \Upsilon_{\omega_{0}}\right)$. This means that $\Psi_{\omega_{0}}$ admits a continuous (respectively, analytic) inverse. Hence, Condition (B2) is established. On the other hand, suppose that $\omega_{1}, \omega_{2} \in \Omega$ with $V_{\omega_{1}} \cap V_{\omega_{2}} \neq \emptyset$. We define two functions $\xi: V_{\omega_{1}} \rightarrow \mathcal{A}\left(\Upsilon_{\omega_{1}}, \Upsilon_{\omega_{1}}\right)$ and $\eta: V_{\omega_{2}} \rightarrow$ $\mathcal{A}\left(\Upsilon_{\omega_{2}}, \Upsilon_{\omega_{2}}\right)$ by

$$
\xi(\omega)(x):=x \oplus_{\omega_{1}} \zeta_{\omega_{1}}(\omega) \quad \text { and } \quad \eta(\omega)(z):=z \oplus_{\omega_{2}}(-1) \odot_{\omega_{2}} \zeta_{\omega_{2}}(\omega) .
$$

Observe that for $a \in \Upsilon_{\omega_{1}}$, if $\chi_{a} \in \mathcal{A}\left(\Upsilon_{\omega_{1}}, \Upsilon_{\omega_{1}}\right)$ is the map given by $\chi_{a}(x):=x \oplus_{\omega_{1}} a$, then $\chi: a \mapsto \chi_{a}$ is continuous and affine (and hence is analytic). Since $\zeta_{\omega_{1}}$ and $\zeta_{\omega_{2}}$ are continuous (respectively, analytic), we know that both $\xi$ and $\eta$, being the compositions of $\zeta_{\omega_{i}}$ with $\chi$, are 
continuous (respectively, analytic). If $\varphi$ is as in Condition (B3), then for any $\omega \in V_{\omega_{1}} \cap V_{\omega_{2}}$ and $x \in \Upsilon_{\omega_{1}}$, one has

$$
\Pi_{2}\left(\Psi_{\omega_{2}}^{-1}\left(\Psi_{\omega_{1}}(\omega, x)\right)\right)=\varphi(\omega)\left(x \oplus_{\omega_{1}} \zeta_{\omega_{1}}(\omega)\right) \oplus_{\omega_{2}}(-1) \odot_{\omega_{2}} \zeta_{\omega_{2}}(\omega)=(\eta(\omega) \circ \varphi(\omega) \circ \xi(\omega))(x) .
$$

Therefore, Condition (B3) still holds when the maps $\Theta_{\omega_{1}}$ and $\Theta_{\omega_{2}}$ are replaced by $\Psi_{\omega_{1}}$ and $\Psi_{\omega_{2}}$, respectively.

Remark 2.4. (a) Suppose that $(\Upsilon, \Omega, \kappa)$ is a locally trivial continuous affine-Banach bundle such that $\Omega$ is paracompact. Then one can see from the discussion before Proposition 4.1 that there always exists a continuous global cross section. This means that when $\Omega$ is paracompact, there is no different between locally trivial continuous affine-Banach bundles over $\Omega$ and locally trivial continuous Banach bundles over $\Omega$.

(b) Note that the " $\mathbb{K}$-analyticity" in the above means the existence of "local power series expansions" (as in Definition 1.6 and p.36 of [32]). We say that a map from an open subset of a $\mathbb{K}$ Banach space to another $\mathbb{K}$-Banach space is $\mathbb{K}$-differentiable if it is Frechet differentiable. One can also defined the notion of locally trivial $\mathbb{K}$-differentiable affine-Banach bundles if one replaces the terms "bi-continuous" and "continuous" in Conditions (B2) and (B3) of Definition 2.2(a) by "KK-bidifferentiable" and "KK-differentiable", respectively. Clearly, a locally trivial $\mathbb{K}$-analytic affine-Banach bundle is a locally trivial $\mathbb{K}$-differentiable affine-Banach bundle. The corresponding statement as Proposition 2.3 is also valid for $\mathbb{K}$-differentiable affine-Banach bundles.

Let us end this section with the following obvious fact for later reference.

Lemma 2.5. If $R \in \mathcal{L}(X)$ satisfying $R^{2}=0$, then $I+R$ is invertible with inverse $I-R$.

\section{3. $\mathcal{J}(X)$ is a LOCALly trivial AnAlytic AFFine-BanaCh BUndLE}

From now on, $X$ is a $\mathbb{K}$-Banach space with $\operatorname{dim}_{\mathbb{K}} X>1$ (could be infinite). Our main concern is the following set

$$
\mathcal{J}(X):=\left\{Q \in \mathcal{L}(X) \backslash\{0, I\}: Q^{2}=Q\right\},
$$

with different structures induced from $\mathcal{L}(X)$. For any $Q \in \mathcal{J}(X)$, it is obvious that both $Q(X)$ and $\operatorname{ker} Q$ belongs to $\mathscr{G}(X)$, and that $Q(X) \top \operatorname{ker} Q$ (i.e., they are complement of each other). Conversely, if $E \in \mathscr{G}(X)$ and $F \in \mathcal{C}_{E}$ (see (2.1)), there is a unique element $Q_{E}^{F} \in \mathcal{J}(X)$ with

$$
Q_{E}^{F}(X)=E \quad \text { and } \quad \operatorname{ker} Q_{E}^{F}=F .
$$

For every $F_{0} \in \mathscr{G}(X)$, let us denote

$$
\mathcal{J}(X)^{F_{0}}:=\left\{Q \in \mathcal{J}(X): \operatorname{ker} Q=F_{0}\right\}, \quad \mathcal{J}(X)_{F_{0}}:=\left\{Q \in \mathcal{J}(X): Q(X)=F_{0}\right\}
$$

as well as

$$
\mathcal{L}^{F_{0}}\left(X, F_{0}\right):=\left\{T \in \mathcal{L}(X): T(X) \subseteq F_{0} \text { and } T\left(F_{0}\right)=(0)\right\} .
$$

The starting point of this paper is the following easy observation.

Lemma 3.1. Let $X$ be a Banach space and $E_{0}, F_{0} \in \mathscr{G}(X)$ with $E_{0} \top F_{0}$. Then $\mathcal{J}(X)^{F_{0}}=\mathcal{L}^{F_{0}}\left(X, F_{0}\right)+$ $Q_{E_{0}}^{F_{0}}$, and $\mathcal{J}(X)_{F_{0}}=\mathcal{L}^{F_{0}}\left(X, F_{0}\right)+Q_{F_{0}}^{E_{0}}$.

Proof. By considering the bijection $Q \mapsto I-Q$ from $\mathcal{J}(X)^{F_{0}}$ onto $\mathcal{J}(X)_{F_{0}}$, one only needs to verify the first equality. In fact, if $E \in \mathcal{C}_{F_{0}}$ (see (2.1)), then

$$
\left(Q_{E}^{F_{0}}-Q_{E_{0}}^{F_{0}}\right)(x)=Q_{E}^{F_{0}}(x)-x=-Q_{F_{0}}^{E}(x) \in F_{0} \quad\left(x \in E_{0}\right) .
$$

From this, as well as the fact that $Q_{E}^{F_{0}}-Q_{E_{0}}^{F_{0}}$ vanish on $F_{0}$, we obtain $\left(Q_{E}^{F_{0}}-Q_{E_{0}}^{F_{0}}\right)(X) \subseteq F_{0}$. Hence, $Q-Q_{E_{0}}^{F_{0}} \in \mathcal{L}^{F_{0}}\left(X, F_{0}\right)$, for every $Q \in \mathcal{J}(X)^{F_{0}}$. 
Conversely, consider an element $R \in \mathcal{L}^{F_{0}}\left(X, F_{0}\right)$. It is not hard to check that $(I+R)\left(E_{0}\right) \in \mathcal{C}_{F_{0}}$. For any $x \in E_{0}$, as $R(x) \in F_{0}$, one has

which gives

$$
Q_{(I+R)\left(E_{0}\right)}^{F_{0}}(x)=Q_{(I+R)\left(E_{0}\right)}^{F_{0}}(x+R(x))=x+R(x),
$$

$$
R(x)=Q_{(I+R)\left(E_{0}\right)}^{F_{0}}(x)-Q_{E_{0}}^{F_{0}}(x) .
$$

Furthermore, as $R, Q_{(I+R)\left(E_{0}\right)}^{F_{0}}$ and $Q_{E_{0}}^{F_{0}}$ all vanish on $F_{0}$, we know that $R=Q_{(I+R)\left(E_{0}\right)}^{F_{0}}-Q_{E_{0}}^{F_{0}}$.

Consider $F_{0} \in \mathcal{C}_{E_{0}}$ and $E_{0}, E_{1} \in \mathcal{C}_{F_{0}}$. We have

$$
Q_{E_{1}}^{F_{0}}\left(E_{0}\right)=Q_{E_{1}}^{F_{0}}\left(E_{0}+F_{0}\right)=E_{1} .
$$

Moreover, Lemma 3.1 allows us to define a map $\pi_{E_{0}, F_{0}}$ from $\mathcal{C}_{F_{0}}$ onto $\mathcal{L}^{F_{0}}\left(X, F_{0}\right)$ via

$$
\pi_{E_{0}, F_{0}}(E):=Q_{E}^{F_{0}}-Q_{E_{0}}^{F_{0}} \quad\left(E \in \mathcal{C}_{F_{0}}\right) .
$$

Set $T:=p_{E_{0}, F_{0}}\left(E_{1}\right) \in \mathcal{L}\left(E_{0}, F_{0}\right)$ (see (2.2)). Then $E_{1}=(I+T)\left(E_{0}\right)$ (see (2.3)). Thus, applying (3.1) to $R:=T \circ \bar{Q}_{E_{0}}^{F_{0}} \in \mathcal{L}^{F_{0}}\left(X, F_{0}\right)$ (where $\bar{Q}_{E_{0}}^{F_{0}}$ is the idempotent $Q_{E_{0}}^{F_{0}}$ regarding as a map from $X$ to $\left.E_{0}\right)$, we get

In other words,

$$
T(x)=Q_{E_{1}}^{F_{0}}(x)-Q_{E_{0}}^{F_{0}}(x) \quad\left(x \in E_{0}\right) .
$$

where

$$
p_{E_{0}, F_{0}}=\Lambda_{E_{0}, F_{0}} \circ \pi_{E_{0}, F_{0}},
$$

$$
\Lambda_{E_{0}, F_{0}}: \mathcal{L}^{F_{0}}\left(X, F_{0}\right) \rightarrow \mathcal{L}\left(E_{0}, F_{0}\right)
$$

is the Banach space isomorphism given by restrictions. Notice that the inverse of $\Lambda_{E_{0}, F_{0}}$ is given by compositions of elements in $\mathcal{L}\left(E_{0}, F_{0}\right)$ with the map $\bar{Q}_{E_{0}}^{F_{0}}: X \rightarrow E_{0}$ as in the above. Furthermore, (3.3) implies

$$
\pi_{E_{0}, F_{0}}\left(E_{1}\right)=-\pi_{E_{1}, F_{0}}\left(E_{0}\right) .
$$

On the other hand, by Relation (3.4), the analytic atlas of $\mathscr{G}(X)$ as in (2.4) can be rewritten as:

$$
\left\{\left(\mathcal{C}_{F_{0}}, \pi_{E_{0}, F_{0}}, \mathcal{L}^{F_{0}}\left(X, F_{0}\right)\right): E_{0}, F_{0} \in \mathscr{G}(X) ; F_{0} \top E_{0}\right\} .
$$

One good point of this atlas is that elements in $\mathcal{L}^{F_{0}}\left(X, F_{0}\right)$ are nilpotent operators of degree two, and Lemma 2.5 applies to them. Another benefit of this atlas is the following result, which gives a clear picture of the topology on $\mathscr{G}(X)$. In particular, we know that $E_{k}$ converges to $E_{0}$ is basically the same as $Q_{E_{k}}^{F_{0}}(x)$ converges to $Q_{E_{0}}^{F_{0}}(x)$ in a uniform way on all bounded subsets.

Corollary 3.2. Suppose that $\left\{E_{k}\right\}_{k \in \mathbb{N}}$ is a sequence in $\mathscr{G}(X)$ and $E_{0} \in \mathscr{G}(X)$. Then $E_{k} \rightarrow E_{0}$ if and only if for every $F_{0} \in \mathfrak{C}_{E_{0}}$ (equivalently, there exists $F_{0} \in \mathfrak{C}_{E_{0}}$ ), there exists $k_{0} \in \mathbb{N}$ such that $E_{k} \in \mathcal{C}_{F_{0}}$ when $k \geq k_{0}$ and that $\left\|Q_{E_{k}}^{F_{0}}-Q_{E_{0}}^{F_{0}}\right\| \rightarrow 0$.

Let $\kappa: \mathcal{J}(X) \rightarrow \mathscr{G}(X)$ be the surjection as given in (1.1). Lemma 3.1 tells us that the fiber $\kappa^{-1}(E)$ (which coincides with $\mathcal{J}(X)_{E}$ ) is an affine-Banach subspace of $\mathcal{L}(X)$, for every $E \in \mathscr{G}(X)$. The main result of this section is that under these affine-Banach space structures on the fibers and the Banach submanifold structure on $\mathcal{J}(X)$ induced from $\mathcal{L}(X),(\mathcal{J}(X), \mathscr{G}(X), \kappa)$ is a locally trivial analytic affine-Banach bundle.

For the proof of this statement, we need to consider the canonical actions of $G \mathcal{L}(X)$ on $\mathcal{J}(X)$ and on $\mathscr{G}(X)$. Indeed, for any $W \in G \mathcal{L}(X)$ and $E, F \in \mathscr{G}(X)$ with $F \top E$, one easily sees that

$$
\operatorname{Ad}(W)\left(Q_{E}^{F}\right):=W \circ Q_{E}^{F} \circ W^{-1}=Q_{W(E)}^{W(F)},
$$

and this produces an action of $G \mathcal{L}(X)$ on $\mathcal{J}(X)$. On the other hand,

$$
\alpha(W, E):=W(E)
$$

induces an action of $G \mathcal{L}(X)$ on $\mathscr{G}(X)$. It is well-known that the two actions $\alpha$ and Ad are $\mathbb{K}$-analytic.

We also need the following easy fact for the proof of the main theorem. 
Lemma 3.3. Let $Y$ be a $\mathbb{K}$-Banach space, and let $A, B \subseteq Y$ be two $\mathbb{K}$-affine-Banach subspaces. Suppose that $B-b_{0} \in \mathscr{G}(Y)$ for an element $b_{0} \in B$. Then there is a continuous affine map $\Gamma: \mathcal{L}(Y) \rightarrow \mathcal{A}(A, B)$ such that whenever $T \in \mathcal{L}(Y)$ satisfying $T(A) \subseteq B$, one has $\Gamma(T)=\left.T\right|_{A}$.

In fact, let us pick an element $D \in \mathcal{C}_{B-b_{0}}$, and define a map $\tilde{Q} \in \mathcal{A}(Y, B)$ by $\tilde{Q}(y):=Q_{B-b_{0}}^{D}(y-$ $\left.b_{0}\right)+b_{0}(y \in Y)$. If we set $\Gamma(T):=\left.\tilde{Q} \circ T\right|_{A}(T \in \mathcal{L}(Y))$, then clearly, $\Gamma$ is a continuous affine map satisfying the requirement.

A final piece of well-known information that we need is the following. For $E, F \in \mathscr{G}(X)$ with $F \top E$, one has $\mathcal{L}^{E}(X, E) \cap \mathcal{L}^{F}(X, F)=\{0\}$ (since $\left.E+F=X\right)$. We identify $\mathcal{L}^{E}(X, E) \oplus \mathcal{L}^{F}(X, F)$ with the sum of the two subspaces in $\mathcal{L}(X)$. If we define a map $\Delta_{E, F}: \mathcal{L}(X) \rightarrow \mathcal{L}(X)$ by $\Delta_{E, F}(T):=$ $Q_{E}^{F} \circ T \circ Q_{F}^{E}(T \in \mathcal{L}(X))$, then both $\Delta_{E, F}$ and $\Delta_{E, F}+\Delta_{F, E}$ are idempotents with

$$
\Delta_{E, F}(\mathcal{L}(X))=\mathcal{L}^{E}(X, E) \text { and }\left(\Delta_{E, F}+\Delta_{F, E}\right)(\mathcal{L}(X))=\mathcal{L}^{E}(X, E) \oplus \mathcal{L}^{F}(X, F) .
$$

Consequently, both $\mathcal{L}^{E}(X, E)$ and $\mathcal{L}^{E}(X, E) \oplus \mathcal{L}^{F}(X, F)$ are complemented subspaces of $\mathcal{L}(X)$. In the following we will denote elements in $\mathcal{L}^{E}(X, E) \oplus \mathcal{L}^{F}(X, F)$ in either the form $(R, S)$ or $R+S$.

Before presenting the main theorem of this section, let us first give an outline of its proof, and give some remarks.

We will begin by showing that $\kappa$ is continuous. We will then show that $(\mathcal{J}(X), \mathscr{G}(X), \kappa)$ satisfies Conditions (B1) and (B2) of Definition 2.2(a) (i.e., the continuous case). From this, we construct an analytic atlas for $\mathcal{J}(X)$ (see (3.18)). This produces a $\mathbb{K}$-analytic Banach manifold structure on $\mathcal{J}(X)$ compatible with the norm topology. We will then verify that the inclusion map from $\mathcal{J}(X)$ to $\mathcal{L}(X)$ is an analytic immersion. Hence, $\mathcal{J}(X)$ is a Banach submanifold of $\mathcal{L}(X)$, under the above Banach manifold structure.

Note that as elements in $\mathcal{J}(X)$ satisfies the algebraic relation $Q^{2}-Q=0$, it is a known fact that $\mathcal{J}(X)$ is a closed submanifold of $\mathcal{L}(X)$ when $\operatorname{dim} X<\infty$. Even though $\mathcal{J}(X)$ being a submanifold of $\mathcal{L}(X)$ may also be a known fact in the infinite dimensional case, in order to verify that $\mathcal{J}(X)$ is a locally trivial analytic affine-Banach bundle over $\mathscr{G}(X)$, we need to use the explicitly atlas for $\mathcal{J}(X)$ as in (3.18). This atlas will also be needed in the later part of this article. One good feature of this analytic atlas for $\mathcal{J}(X)$ is that it is "algebraic" in nature (see (3.20) and (3.22)).

Finally, we will establish Conditions (B2) and (B3) of Definition 2.2(b) (i.e. the analytic case). Observe that if the fiber over each point in $\mathscr{G}(X)$ were a Banach space, then one might use Proposition 1.2 in Chapter 3 of [27] to simplify the argument. However, since we are in the affine-Banach setting, we give a more direct argument here.

Theorem 3.4. Let $X$ be a $\mathbb{K}$-Banach space. If $\mathcal{J}(X)$ is equipped with the $\mathbb{K}$-analytic Banach submanifold structure induced from $\mathcal{L}(X)$, then $(\mathcal{J}(X), \mathscr{G}(X), \kappa)$ is a locally trivial $\mathbb{K}$-analytic affine-Banach bundle, such that the affine-Banach space structure on $\kappa^{-1}(E)$ is the one induced from $\mathcal{L}(X)$, for every $E \in \mathscr{G}(X)$.

Proof. We first establish the continuity of $\kappa$. For this, let us consider a sequence $\left\{Q_{E_{n}}^{F_{n}}\right\}_{n \in \mathbb{N}}$ in $\mathcal{J}(X)$ converging to $Q_{E_{0}}^{F_{0}} \in \mathcal{J}(X)$; i.e.,

$$
\left\|I-\left(Q_{F_{n}}^{E_{n}}+Q_{E_{0}}^{F_{0}}\right)\right\|=\left\|\left(Q_{E_{n}}^{F_{n}}+Q_{F_{0}}^{E_{0}}\right)-I\right\|=\left\|Q_{E_{n}}^{F_{n}}-Q_{E_{0}}^{F_{0}}\right\| \rightarrow 0 .
$$

As $\mathcal{L}(X)$ is a unital Banach algebra, we know that $Q_{F_{n}}^{E_{n}}+Q_{E_{0}}^{F_{0}}$ and $Q_{E_{n}}^{F_{n}}+Q_{F_{0}}^{E_{0}}$ are eventually invertible, and we assume that they are invertible for all $n \in \mathbb{N}$. The relation $\left(Q_{E_{n}}^{F_{n}}+Q_{F_{0}}^{E_{0}}\right)(X)=X$ and $\operatorname{ker}\left(Q_{F_{n}}^{E_{n}}+Q_{E_{0}}^{F_{0}}\right)=\{0\}$ will then imply that $E_{n} \in \mathcal{C}_{F_{0}}$.

By Corollary 3.2, we need to show that $Q_{E_{n}}^{F_{0}} \rightarrow Q_{E_{0}}^{F_{0}}$. Indeed, it is clear that

$$
Q_{E_{n}}^{F_{n}} \circ Q_{E_{n}}^{F_{0}}=Q_{E_{n}}^{F_{0}} \text { and } Q_{E_{0}}^{F_{0}} \circ Q_{E_{n}}^{F_{0}}=Q_{E_{0}}^{F_{0}} \text {. }
$$

Moreover, one has $\left\|Q_{E_{n}}^{F_{n}}-Q_{E_{0}}^{F_{0}}\right\| \leq 1 / 2$ when $n$ is large, and in this case,

$$
\left\|Q_{E_{n}}^{F_{0}}(x)\right\|=\left\|Q_{E_{n}}^{F_{n}}\left(Q_{E_{n}}^{F_{0}}(x)\right)\right\| \leq\left\|Q_{E_{0}}^{F_{0}}\left(Q_{E_{n}}^{F_{0}}(x)\right)\right\|+\left\|Q_{E_{n}}^{F_{0}}(x)\right\| / 2 \quad(x \in X),
$$


which implies $\left\|Q_{E_{n}}^{F_{0}}\right\| \leq 2\left\|Q_{E_{0}}^{F_{0}}\right\|$. It follows that

$$
\lambda_{0}:=\sup _{n \in \mathbb{N}}\left\|Q_{F_{0}}^{E_{n}}\right\|<\infty .
$$

Now, for any $\epsilon>0$, there is $n_{0}$ such that $\left\|Q_{E_{n}}^{F_{n}}-Q_{E_{0}}^{F_{0}}\right\|<\epsilon$ whenever $n \geq n_{0}$. It then follows from $Q_{E_{0}}^{F_{0}} \circ Q_{F_{0}}^{E_{n}}=0$ and Relation (3.11) that for any $x \in X$,

$$
\left\|Q_{E_{n}}^{F_{n}}(x)-Q_{E_{n}}^{F_{0}}(x)\right\|=\left\|Q_{E_{n}}^{F_{n}}\left(Q_{F_{0}}^{E_{n}}(x)\right)-Q_{E_{0}}^{F_{0}}\left(Q_{F_{0}}^{E_{n}}(x)\right)\right\|<\epsilon \lambda_{0}\|x\| .
$$

Thus, we have $\left\|Q_{E_{n}}^{F_{0}}-Q_{E_{n}}^{F_{n}}\right\| \rightarrow 0$. This, together with $\left\|Q_{E_{n}}^{F_{n}}-Q_{E_{0}}^{F_{0}}\right\| \rightarrow 0$, implies the required convergence, and hence $\kappa$ is continuous.

On the other hand, it follows from Lemma 3.1 that $\kappa^{-1}(E)$ is an affine-Banach subspace of $\mathcal{L}(X)$, for each $E \in \mathscr{G}(X)$; in other words, Condition (B1) in Definition 2.2(a) is satisfied.

Let us now show that $(\mathcal{J}(X), \mathscr{G}(X), \kappa)$ satisfies Condition (B2) of Definition 2.2(a). We will do this via the construction of an analytic (and hence continuous) local right inverse for the evaluation maps from $G \mathcal{L}(X)$ to orbits of the action $\alpha$ as in (3.9). For this, we fix arbitrary elements $E_{0}, F_{0} \in \mathscr{G}(X)$ with $E_{0} \top F_{0}$. Consider $E \in \mathcal{C}_{F_{0}}$. Since $Q_{E}^{F_{0}}-Q_{E_{0}}^{F_{0}} \in \mathcal{L}^{F_{0}}\left(X, F_{0}\right)$, we know from Lemma 2.5 that $\left(I+\left(Q_{E}^{F_{0}}-Q_{E_{0}}^{F_{0}}\right)\right)^{-1}=I-\left(Q_{E}^{F_{0}}-Q_{E_{0}}^{F_{0}}\right)$. Therefore, if we set

$$
\Xi_{E_{0}, F_{0}}(E):=Q_{E}^{F_{0}}+Q_{F_{0}}^{E_{0}},
$$

then $\Xi_{E_{0}, F_{0}}(E) \in G \mathcal{L}(X)$ and

$$
\Xi_{E_{0}, F_{0}}(E)^{-1}=Q_{F_{0}}^{E}+Q_{E_{0}}^{F_{0}}=2 I-\Xi_{E_{0}, F_{0}}(E) .
$$

Recall that $\left(\mathcal{C}_{F_{0}}, \pi_{E_{0}, F_{0}}, \mathcal{L}^{F_{0}}\left(X, F_{0}\right)\right)$ is a local chart for $\mathscr{G}(X)$ near $E_{0}$ (see (3.7)). As

$$
\Xi_{E_{0}, F_{0}}\left(\pi_{E_{0}, F_{0}}^{-1}(R)\right)=I+R \quad\left(R \in \mathcal{L}^{F_{0}}\left(X, F_{0}\right)\right),
$$

the map $\Xi_{E_{0}, F_{0}}: \mathcal{C}_{F_{0}} \rightarrow G \mathcal{L}(X)$ is analytic. Moreover, one has

$$
\alpha\left(\Xi_{E_{0}, F_{0}}(E), E_{0}\right)=Q_{E}^{F_{0}}\left(E_{0}\right)=Q_{E}^{F_{0}}(X)=E .
$$

Consequently, $\Xi_{E_{0}, F_{0}}$ is an analytic local right inverse for the evaluation map at $E_{0}$ from $G \mathcal{L}(X)$ to the orbit $\alpha\left(G \mathcal{L}(X), E_{0}\right)$.

We now define $\Theta_{E_{0}, F_{0}}: \mathcal{C}_{F_{0}} \times \mathfrak{J}(X)_{E_{0}} \rightarrow \kappa^{-1}\left(\mathcal{C}_{F_{0}}\right)$ by

$$
\Theta_{E_{0}, F_{0}}(E, Q):=\operatorname{Ad}\left(\Xi_{E_{0}, F_{0}}(E)\right)(Q) \quad\left(E \in \mathcal{C}_{F_{0}}, Q \in \mathcal{J}(X)_{E_{0}}\right) .
$$

For $E \in \mathcal{C}_{F_{0}}$ and $F \in \mathcal{C}_{E_{0}}$, we know from (3.8) and (3.15) that

$$
\Theta_{E_{0}, F_{0}}\left(E, Q_{E_{0}}^{F}\right)=Q_{\Xi_{E_{0}, F_{0}}(E)\left(E_{0}\right)}^{\Xi_{E_{0}, F_{0}}(E)(F)}=Q_{E}^{\left(Q_{E}^{F_{0}}+Q_{F_{0}}^{E_{0}}\right)(F)} .
$$

This implies that $\Theta_{E_{0}, F_{0}}$ is well-defined and injective (since $\Xi_{E_{0}, F_{0}}(E)$ is invertible). Furthermore, it is clear from the definition that $\Theta_{E_{0}, F_{0}}$ is fiberwise affine. On the other hand, for any $E^{\prime} \in \mathcal{C}_{F_{0}}$ and $F^{\prime} \in \mathcal{C}_{E^{\prime}}$, if we set $F^{\prime \prime}:=\Xi_{E_{0}, F_{0}}\left(E^{\prime}\right)^{-1}\left(F^{\prime}\right)$, then $F^{\prime \prime} \in \mathcal{C}_{E_{0}}$ because $\operatorname{Ad}\left(\Xi_{E_{0}, F_{0}}\left(E^{\prime}\right)^{-1}\right)\left(Q_{E^{\prime}}^{F^{\prime}}\right)=Q_{E_{0}}^{F^{\prime \prime}}$, and so, $\Theta_{E_{0}, F_{0}}\left(E^{\prime}, Q_{E_{0}}^{F^{\prime \prime}}\right)=Q_{E^{\prime}}^{F^{\prime}}$. This means that $\Theta_{E_{0}, F_{0}}$ is surjective.

In the following, we establish the bi-continuity of $\Theta_{E_{0}, F_{0}}$. Let $\left\{F_{n}\right\}_{n \in \mathbb{N}}$ and $\left\{E_{n}\right\}_{n \in \mathbb{N}}$ be sequences in $\mathcal{C}_{E_{0}}$ and in $\mathcal{C}_{F_{0}}$, respectively. If $\left\{E_{n}\right\}_{n \in \mathbb{N}}$ converges to $E \in \mathcal{C}_{F_{0}}$ and $\left\{Q_{E_{0}}^{F_{n}}\right\}_{n \in \mathbb{N}}$ converges to $Q_{E_{0}}^{F} \in \mathcal{J}(X)_{E_{0}}$, then it follows from the continuity of $\Xi_{E_{0}, F_{0}}$ and (3.13) that

$$
\Theta_{E_{0}, F_{0}}\left(E_{n}, Q_{E_{0}}^{F_{n}}\right)=\Xi_{E_{0}, F_{0}}\left(E_{n}\right) Q_{E_{0}}^{F_{n}}\left(2 I-\Xi_{E_{0}, F_{0}}\left(E_{n}\right)\right) \rightarrow \Theta_{E_{0}, F_{0}}\left(E, Q_{E_{0}}^{F}\right) .
$$

Conversely, assume that $\Theta_{E_{0}, F_{0}}\left(E_{n}, Q_{E_{0}}^{F_{n}}\right) \rightarrow \Theta_{E_{0}, F_{0}}\left(E, Q_{E_{0}}^{F}\right)$. The continuity of $\kappa$ and Relation (3.17) give $E_{n} \rightarrow E$ and $\Xi_{E_{0}, F_{0}}\left(E_{n}\right)\left(F_{n}\right) \rightarrow \Xi_{E_{0}, F_{0}}(E)(F)$. Consequently, the continuity of $\Xi_{E_{0}, F_{0}}$ tells us that

$$
F_{n}=\left(2 I-\Xi_{E_{0}, F_{0}}\left(E_{n}\right)\right)\left(\Xi_{E_{0}, F_{0}}\left(E_{n}\right)\left(F_{n}\right)\right) \rightarrow F .
$$

From this, we know that $Q_{E_{0}}^{F_{n}} \rightarrow Q_{E_{0}}^{F}$. 
We are now ready to construct a Banach manifold structure on $\mathcal{J}(X)$ that is compatible with the norm topology. For every $E_{0} \in \mathscr{G}(X)$ and $F_{0} \in \mathcal{C}_{E_{0}}$, we consider the bijection $\phi_{E_{0}, F_{0}}: \mathcal{J}(X)_{E_{0}} \rightarrow$ $\mathcal{L}^{E_{0}}\left(X, E_{0}\right)$ induced by Lemma 3.1 ; namely,

$$
\phi_{E_{0}, F_{0}}(Q):=Q-Q_{E_{0}}^{F_{0}} \quad\left(Q \in \mathcal{J}(X)_{E_{0}}\right) .
$$

Set $\mu_{E_{0}, F_{0}}: \kappa^{-1}\left(\mathcal{C}_{F_{0}}\right) \rightarrow \mathcal{L}^{F_{0}}\left(X, F_{0}\right) \oplus \mathcal{L}^{E_{0}}\left(X, E_{0}\right)$ to be the map $\left(\pi_{E_{0}, F_{0}} \times \phi_{E_{0}, F_{0}}\right) \circ \Theta_{E_{0}, F_{0}}^{-1}$. We claim that

$$
\left\{\left(\kappa^{-1}\left(\mathcal{C}_{F_{0}}\right), \mu_{E_{0}, F_{0}}, \mathcal{L}^{F_{0}}\left(X, F_{0}\right) \oplus \mathcal{L}^{E_{0}}\left(X, E_{0}\right)\right): E_{0}, F_{0} \in \mathscr{G}(X) ; F_{0} \top E_{0}\right\}
$$

is an analytic atlas for $\mathcal{J}(X)$.

In fact, $\mu_{E_{0}, F_{0}}$ is a homeomorphism since $\pi_{E_{0}, F_{0}}, \phi_{E_{0}, F_{0}}$ and $\Theta_{E_{0}, F_{0}}$ are homeomorphisms. Notice also that if $E \in \mathcal{C}_{F_{0}}$ and $F \in \mathcal{C}_{E}$, then we have

$$
\begin{aligned}
\mu_{E_{0}, F_{0}}\left(Q_{E}^{F}\right) & =\left(Q_{E}^{F_{0}}-Q_{E_{0}}^{F_{0}}, \operatorname{Ad}\left(Q_{F_{0}}^{E}+Q_{E_{0}}^{F_{0}}\right)\left(Q_{E}^{F}\right)-Q_{E_{0}}^{F_{0}}\right) \\
& =\left(Q_{E}^{F_{0}}-Q_{E_{0}}^{F_{0}}, Q_{E_{0}}^{F_{0}} Q_{E}^{F} Q_{F_{0}}^{E_{0}}\right),
\end{aligned}
$$

because of (3.2), (3.11) and (3.17). Moreover, for $\left.(R, S) \in \mathcal{L}^{F_{0}}\left(X, F_{0}\right) \oplus \mathcal{L}^{E_{0}}\left(X, E_{0}\right)\right)$, one has

$$
\begin{aligned}
\mu_{E_{0}, F_{0}}^{-1}(R, S) & =(I+R)\left(S+Q_{E_{0}}^{F_{0}}\right)(I-R) \\
& =S-S R+Q_{E_{0}}^{F_{0}}+R S-R S R+R,
\end{aligned}
$$

because of (3.14), Lemma 2.5 as well as the facts that $R(X) \subseteq F_{0}$ and $R Q_{E_{0}}^{F_{0}}=R$.

Assume now that $E_{1}, F_{1} \in \mathscr{G}(X)$ with $E_{1} \top F_{1}$ such that $\kappa^{-1}\left(\mathcal{C}_{F_{0}}\right) \cap \kappa^{-1}\left(\mathcal{C}_{F_{1}}\right) \neq \emptyset$. Consider an arbitrary element $(R, S) \in \mu_{E_{0}, F_{0}}\left(\kappa^{-1}\left(\mathcal{C}_{F_{0}} \cap \mathcal{C}_{F_{1}}\right)\right)$. By Lemma 3.1, there exist unique elements $E_{R} \in \mathcal{C}_{F_{0}}$ with $R=Q_{E_{R}}^{F_{0}}-Q_{E_{0}}^{F_{0}}$. These produce, via (3.19) as well as (3.21),

$$
\mu_{E_{1}, F_{1}}\left(\mu_{E_{0}, F_{0}}^{-1}(R, S)\right)=\left(Q_{E_{R}}^{F_{1}}-Q_{E_{1}}^{F_{1}}, \operatorname{Ad}\left(Q_{F_{1}}^{E_{R}}+Q_{E_{1}}^{F_{1}}\right)\left(\operatorname{Ad}(I+R)\left(S+Q_{E_{0}}^{F_{0}}\right)\right)-Q_{E_{1}}^{F_{1}}\right) .
$$

Since $\left\{\left(\mathcal{C}_{F}, \pi_{E, F}, \mathcal{L}^{F}(X, F)\right): E, F \in \mathscr{G}(X) ; F \top E\right\}$ is an analytic atlas of $\mathscr{G}(X)$, the map from $\pi_{E_{0}, F_{0}}\left(\mathcal{C}_{F_{0}} \cap \mathcal{C}_{F_{1}}\right)$ onto $\pi_{E_{1}, F_{1}}\left(\mathcal{C}_{F_{0}} \cap \mathcal{C}_{F_{1}}\right)$ that sends $Q_{E}^{F_{0}}-Q_{E_{0}}^{F_{0}}$ to $Q_{E}^{F_{1}}-Q_{E_{1}}^{F_{1}}$ is analytic. In other words, if we set

$$
\Phi(R):=Q_{E_{R}}^{F_{1}}-Q_{E_{1}}^{F_{1}},
$$

then $(R, S) \mapsto \Phi(R)$ is an analytic map from $\pi_{E_{0}, F_{0}}\left(\mathcal{C}_{F_{0}} \cap \mathcal{C}_{F_{1}}\right) \times \mathcal{L}^{E_{0}}\left(X, E_{0}\right)$ to $\pi_{E_{1}, F_{1}}\left(\mathcal{C}_{F_{0}} \cap \mathcal{C}_{F_{1}}\right)$. Thus, the assignment

$$
(R, S) \mapsto(I-\Phi(R))(I+R)\left(S+Q_{E_{0}}^{F_{0}}\right)(I-R)(I+\Phi(R))-Q_{E_{1}}^{F_{1}}
$$

is also analytic. Consequently, $\mu_{E_{1}, F_{1}} \circ \mu_{E_{0}, F_{0}}^{-1}$ is analytic on $\mu_{E_{0}, F_{0}}\left(\mathcal{C}_{F_{0}} \cap \mathcal{C}_{F_{1}}\right)$, and (3.18) is an analytic atlas for $\mathcal{J}(X)$.

Next, we will show that, when equipped with the above manifold structure, $\mathcal{J}(X)$ is a Banach submanifold of $\mathcal{L}(X)$, by verifying that the inclusion map $\iota: \mathcal{J}(X) \rightarrow \mathcal{L}(X)$ is an analytic immersion. Let us fix $E_{0} \in \mathscr{G}(X)$ and $F_{0} \in \mathcal{C}_{E_{0}}$. Set $\theta_{E_{0}, F_{0}}$ to be the map $\iota \circ \mu_{E_{0}, F_{0}}^{-1}: \mathcal{L}^{F_{0}}\left(X, F_{0}\right) \oplus \mathcal{L}^{E_{0}}\left(X, E_{0}\right) \rightarrow$ $\mathcal{L}(X)$. By (3.22), one has

$$
\theta_{E_{0}, F_{0}}(R, S)=S-S R+Q_{E_{0}}^{F_{0}}+R S-R S R+R
$$

Hence, $\iota$ is analytic. Consider

$$
\mathbf{T}(\iota): \mathbf{T}(\mathcal{J}(X)) \rightarrow \mathbf{T}(\mathcal{L}(X))
$$

to be the map between the respective tangent bundles induced by $\iota$. We need to show that the map

$$
\mathbf{T}_{Q_{E_{0}}^{F_{0}}}(\iota)=\theta_{E_{0}, F_{0}}^{\prime}(0,0)
$$

(here, $\theta_{E_{0}, F_{0}}^{\prime}$ is the derivative of $\left.\theta_{E_{0}, F_{0}}\right)$ will send $\mathcal{L}^{F_{0}}\left(X, F_{0}\right) \oplus \mathcal{L}^{E_{0}}\left(X, E_{0}\right)$ bijectively onto a complemented subspace of $\mathcal{L}(X)$. 
As $\mathcal{L}^{F_{0}}\left(X, F_{0}\right) \oplus \mathcal{L}^{E_{0}}\left(X, E_{0}\right)$ is already a complemented subspace of $\mathcal{L}(X)$, this claim is established if one can show that $\theta_{E_{0}, F_{0}}^{\prime}(0,0)$ is the inclusion map. To see this, we observe that for every $\epsilon \in(0,1)$ and $(R, S) \in \mathcal{L}^{F_{0}}\left(X, F_{0}\right) \oplus \mathcal{L}^{E_{0}}\left(X, E_{0}\right)$, with $\|R\|+\|S\|<\epsilon$, one has, via (3.23),

$$
\left\|\theta_{E_{0}, F_{0}}(R, S)-\theta_{E_{0}, F_{0}}(0,0)-(R+S)\right\|=\|R S-S R-R S R\|<\epsilon^{2}(2+\epsilon) .
$$

This gives $\theta_{E_{0}, F_{0}}^{\prime}(0,0)(R, S)=R+S$, as required.

Finally, we will establish that $(\mathcal{J}(X), \mathscr{G}(X), \kappa)$ is a locally trivial $\mathbb{K}$-analytic affine-Banach bundle. Indeed, as $\pi_{E_{0}, F_{0}} \circ \kappa \circ \mu_{E_{0}, F_{0}}^{-1}(R, S)=R$, we know that $\kappa: \mathcal{J}(X) \rightarrow \mathscr{G}(X)$ is an analytic. Moreover, since the definition of the analytic atlas as in (3.18) is defined via the map $\Theta_{E_{0}, F_{0}}$ as well as the bi-analytic maps $\pi_{E_{0}, F_{0}}$ and $\phi_{E_{0}, F_{0}}$, it is a tautology that $\Theta_{E_{0}, F_{0}}$ is bi-analytic; i.e., Condition (B2) of Definition 2.2(b) holds.

Suppose that $\varphi$ is the map as in Condition (B3) for $\Theta_{E_{1}, F_{1}}^{-1} \circ \Theta_{E_{0}, F_{0}}$. It follows from (3.14) and Lemma 2.5 that for any $E \in \mathcal{C}_{F_{0}} \cap \mathcal{C}_{F_{1}}$ and $Q \in \mathcal{J}(X)_{E_{0}}$, one has

$$
\begin{aligned}
\varphi(E)(Q) & =\operatorname{Ad}\left(\Xi_{E_{1}, F_{1}}(E)^{-1}\right) \circ \operatorname{Ad}\left(\Xi_{E_{0}, F_{0}}(E)\right)(Q) \\
& =\left(I-\pi_{E_{1}, F_{1}}(E)\right)\left(I+\pi_{E_{0}, F_{0}}(E)\right) Q\left(I-\pi_{E_{0}, F_{0}}(E)\right)\left(I+\pi_{E_{1}, F_{1}}(E)\right) .
\end{aligned}
$$

Let us define $\psi: \mathcal{C}_{F_{0}} \cap \mathcal{C}_{F_{1}} \rightarrow \mathcal{L}(\mathcal{L}(X))$ by

$$
\psi(E)(T)=\operatorname{Ad}\left(I-\pi_{E_{1}, F_{1}}(E)\right) \circ \operatorname{Ad}\left(I+\pi_{E_{0}, F_{0}}(E)\right)(T) \quad(T \in \mathcal{L}(X)) .
$$

Since both $\pi_{E_{0}, F_{0}}$ and $\pi_{E_{1}, F_{1}}$ are analytic, we know that the map $\psi$ is analytic. On the other hand, as $\mathcal{L}^{E_{1}}\left(X, E_{1}\right) \in \mathscr{G}(\mathcal{L}(X))$, we obtain a continuous affine map

$$
\Gamma: \mathcal{L}(\mathcal{L}(X)) \rightarrow \mathcal{A}\left(\mathcal{J}(X)_{E_{0}}, \mathcal{J}(X)_{E_{1}}\right)
$$

satisfying the condition in Lemma 3.3. It follows that

$$
\Gamma(\psi(E))=\left.\psi(E)\right|_{\mathcal{J}(X)_{E_{0}}}=\varphi(E) \quad\left(E \in \mathcal{C}_{F_{0}} \cap \mathcal{C}_{F_{1}}\right) .
$$

Since $\Gamma \circ \psi$ is an analytic map from $\mathcal{C}_{F_{0}} \cap \mathcal{C}_{F_{1}}$ to $\mathcal{A}\left(\mathcal{J}(X)_{E_{0}}, \mathcal{J}(X)_{E_{1}}\right)$, Condition (B3) of Definition $2.2(\mathrm{~b})$ is satisfied.

Example 3.5. We equip $\mathbb{C}^{2}$ with the usual Euclidean norm. For $\lambda \in \mathbb{C}$, we set $E_{\lambda}:=\left\{\left[\begin{array}{c}a \\ \lambda a\end{array}\right]: a \in \mathbb{C}\right\}$ and $E_{\infty}:=\left\{\left[\begin{array}{l}0 \\ b\end{array}\right]: b \in \mathbb{C}\right\}$. Then $\mathscr{G}\left(\mathbb{C}^{2}\right)=\left\{E_{\lambda}: \lambda \in \mathbb{C} \cup\{\infty\}\right\}$ and

$$
\mathcal{J}\left(\mathbb{C}^{2}\right)=\left\{\left[\begin{array}{ll}
1 & \gamma \\
0 & 0
\end{array}\right]: \gamma \in \mathbb{C}\right\} \cup\left\{\left[\begin{array}{cc}
1-\alpha & \alpha / \lambda \\
\lambda(1-\alpha) & \alpha
\end{array}\right]: \alpha, \lambda \in \mathbb{C} ; \lambda \neq 0\right\} \cup\left\{\left[\begin{array}{ll}
0 & 0 \\
\delta & 1
\end{array}\right]: \delta \in \mathbb{C}\right\} .
$$

Notice also that $E_{0} \top E_{\infty}, Q_{E_{0}}^{E_{\infty}}=\left[\begin{array}{ll}1 & 0 \\ 0 & 0\end{array}\right]$ and $Q_{E_{\infty}}^{E_{0}}=\left[\begin{array}{ll}0 & 0 \\ 0 & 1\end{array}\right]$. Moreover, for each $\lambda \in \mathbb{C} \backslash\{0\}$, we have $E_{\lambda} \top E_{\infty}, E_{\lambda} \top E_{0}, Q_{E_{\lambda}}^{E_{\infty}}=\left[\begin{array}{ll}1 & 0 \\ \lambda & 0\end{array}\right]$ as well as $Q_{E_{\lambda}}^{E_{0}}=\left[\begin{array}{cc}0 & 1 / \lambda \\ 0 & 1\end{array}\right]$.

On the other hand, $\mathcal{J}\left(\mathbb{C}^{2}\right)_{E_{0}}=\left\{\left[\begin{array}{ll}1 & \gamma \\ 0 & 0\end{array}\right]: \gamma \in \mathbb{C}\right\}, \mathcal{J}\left(\mathbb{C}^{2}\right)_{E_{\infty}}=\left\{\left[\begin{array}{ll}0 & 0 \\ \delta & 1\end{array}\right]: \delta \in \mathbb{C}\right\}$,

$$
\mathcal{L}^{E_{\infty}}\left(\mathbb{C}^{2}, E_{\infty}\right)=\left\{\left[\begin{array}{ll}
0 & 0 \\
\delta & 0
\end{array}\right]: \delta \in \mathbb{C}\right\} \quad \text { and } \quad \mathcal{L}^{E_{0}}\left(\mathbb{C}^{2}, E_{0}\right)=\left\{\left[\begin{array}{ll}
0 & \gamma \\
0 & 0
\end{array}\right]: \gamma \in \mathbb{C}\right\} .
$$

Furthermore, for $\lambda \in \mathbb{C} \backslash\{0\}$, one has $\mathcal{J}\left(\mathbb{C}^{2}\right)_{E_{\lambda}}=\left\{\left[\begin{array}{cc}1-\alpha & \alpha / \lambda \\ \lambda(1-\alpha) & \alpha\end{array}\right]: \alpha \in \mathbb{C}\right\}$ and

$$
\mathcal{L}^{E_{\lambda}}\left(\mathbb{C}, E_{\lambda}\right)=\left\{\alpha\left[\begin{array}{cc}
-1 & 1 / \lambda \\
-\lambda & 1
\end{array}\right]: \alpha \in \mathbb{C}\right\} .
$$

The map $\pi_{E_{0}, E_{\infty}}: \mathcal{C}_{E_{\infty}}=\left\{E_{\lambda}: \lambda \in \mathbb{C}\right\} \rightarrow \mathcal{L}^{E_{\infty}}\left(\mathbb{C}^{2}, E_{\infty}\right)$ is given by

$$
\pi_{E_{0}, E_{\infty}}\left(E_{\lambda}\right)=\left[\begin{array}{ll}
0 & 0 \\
\lambda & 0
\end{array}\right] \quad(\lambda \in \mathbb{C}) .
$$


We also have $\mathcal{C}_{E_{0}}=\left\{E_{\lambda}: \lambda \in \mathbb{C} \backslash\{0\}\right\} \cup\left\{E_{\infty}\right\}$. The map $\pi_{E_{\infty}, E_{0}}: \mathcal{C}_{E_{0}} \rightarrow \mathcal{L}^{E_{0}}\left(\mathbb{C}^{2}, E_{0}\right)$ is given by

$$
\pi_{E_{\infty}, E_{0}}\left(E_{\infty}\right)=\left[\begin{array}{ll}
0 & 0 \\
0 & 0
\end{array}\right] \quad \text { and } \quad \pi_{E_{\infty}, E_{0}}\left(E_{\lambda}\right)=\left[\begin{array}{cc}
0 & 1 / \lambda \\
0 & 0
\end{array}\right] \quad(\lambda \in \mathbb{C} \backslash\{0\}) .
$$

Consider $\lambda \in \mathbb{C}$. If $\lambda \neq 0$, then $\mu_{E_{0}, E_{\infty}}: \kappa^{-1}\left(E_{\lambda}\right) \rightarrow \mathcal{L}^{E_{\infty}}\left(\mathbb{C}^{2}, E_{\infty}\right) \oplus \mathcal{L}^{E_{0}}\left(\mathbb{C}^{2}, E_{0}\right)$ is given by

$$
\mu_{E_{0}, E_{\infty}}\left(\left[\begin{array}{cc}
1-\alpha & \alpha / \lambda \\
\lambda(1-\alpha) & \alpha
\end{array}\right]\right)=\left(\left[\begin{array}{ll}
0 & 0 \\
\lambda & 0
\end{array}\right],\left[\begin{array}{cc}
0 & \alpha / \lambda \\
0 & 0
\end{array}\right]\right) .
$$

In the case when $\lambda=0$, the map $\mu_{E_{0}, E_{\infty}}: \kappa^{-1}\left(E_{0}\right) \rightarrow \mathcal{L}^{E_{\infty}}\left(\mathbb{C}^{2}, E_{\infty}\right) \oplus \mathcal{L}^{E_{0}}\left(\mathbb{C}^{2}, E_{0}\right)$ is given by $\mu_{E_{0}, E_{\infty}}\left(\left[\begin{array}{ll}1 & \gamma \\ 0 & 0\end{array}\right]\right)=\left(\left[\begin{array}{ll}0 & 0 \\ 0 & 0\end{array}\right],\left[\begin{array}{ll}0 & \gamma \\ 0 & 0\end{array}\right]\right)$.

On the other extreme, the map $\mu_{E_{\infty}, E_{0}}: \kappa^{-1}\left(\mathcal{C}_{E_{0}}\right) \rightarrow \mathcal{L}^{E_{0}}\left(\mathbb{C}^{2}, E_{0}\right) \oplus \mathcal{L}^{E_{\infty}}\left(\mathbb{C}^{2}, E_{\infty}\right)$ is given by

$$
\mu_{E_{\infty}, E_{0}}\left(\left[\begin{array}{cc}
1-\alpha & \alpha / \lambda \\
\lambda(1-\alpha) & \alpha
\end{array}\right]\right)=\left(\left[\begin{array}{cc}
0 & 1 / \lambda \\
0 & 0
\end{array}\right],\left[\begin{array}{cc}
0 & 0 \\
\lambda(1-\alpha) & 0
\end{array}\right]\right)
$$

and $\mu_{E_{\infty}, E_{0}}\left(\left[\begin{array}{ll}0 & 0 \\ \delta & 1\end{array}\right]\right)=\left(\left[\begin{array}{ll}0 & 0 \\ 0 & 0\end{array}\right],\left[\begin{array}{ll}0 & 0 \\ \delta & 0\end{array}\right]\right)$.

The two charts $\left(\kappa^{-1}\left(\mathcal{C}_{E_{\infty}}\right), \mu_{E_{0}, E_{\infty}}, \mathcal{L}^{E_{\infty}}\left(X, E_{\infty}\right) \oplus \mathcal{L}^{E_{0}}\left(X, E_{0}\right)\right)$ and

$$
\left(\kappa^{-1}\left(\mathcal{C}_{E_{0}}\right), \mu_{E_{\infty}, E_{0}}, \mathcal{L}^{E_{0}}\left(X, E_{0}\right) \oplus \mathcal{L}^{E_{\infty}}\left(X, E_{\infty}\right)\right)
$$

form an analytic atlas for $\mathcal{J}\left(\mathbb{C}^{2}\right)$, which produces the Banach submanifold structure induced from $M_{2}(\mathbb{C})$.

There is another way to consider fibration of $\mathcal{J}(X)$ over $\mathscr{G}(X)$, namely, through the map $\kappa^{\prime}$ : $\mathcal{J}(X) \rightarrow \mathscr{G}(X)$ that sends $Q$ to $\operatorname{ker} Q$. The same conclusion as in Theorem 3.4 holds for $\left(\mathcal{J}(X), \mathscr{G}(X), \kappa^{\prime}\right)$.

Let us denote by $\mathcal{V}(X)$ the set of "self-inverse mappings"; i.e.

$$
\mathcal{V}(X):=\left\{V \in G \mathcal{L}(X) \backslash\{I,-I\}: V^{2}=I\right\} .
$$

For each $V \in \mathcal{V}(X)$, let us denote $X^{V}:=\{x \in X: V(x)=x\}$, and set $\bar{\kappa}: \mathcal{V}(X) \rightarrow \mathscr{G}(X)$ to be the map given by $\bar{\kappa}(V):=X^{V}$. Since the bi-analytic bijection $T \mapsto 2 T-I$ sends $\mathcal{J}(X)$ onto $\mathcal{V}(X)$, Theorem 3.4 tells us that $\mathcal{V}(X)$ is a Banach submanifold of $\mathcal{L}(X)$. More precisely, define $\bar{\mu}_{E, F}: \bar{\kappa}^{-1}\left(\mathcal{C}_{F}\right) \rightarrow \mathcal{L}^{F}(X, F) \oplus \mathcal{L}^{E}(X, E)$ by

$$
\bar{\mu}_{E, F}(V):=\left(Q_{\left(I_{X}+V\right)(X)}^{F}-Q_{E}^{F}, Q_{E}^{F} V Q_{F}^{E} / 2\right) \quad\left(V \in \mathcal{V}(X)_{E}\right)
$$

(see (3.20)). Then $\bar{\mu}_{E, F}^{-1}(R, S)=2(R S+R-R S R+S-S R)+Q_{E}^{F}-Q_{F}^{E}$ (see (3.22)), and $\left\{\bar{\kappa}^{-1}\left(\mathcal{C}_{F}\right), \bar{\mu}_{E, F}, \mathcal{L}^{F}(X, F) \oplus \mathcal{L}^{E}(X, E): E, F \in \mathscr{G}(X) ; F \top E\right\}$ is an analytic atlas for the Banach submanifold structure on $\mathcal{V}(X)$ induced from $\mathcal{L}(X)$. Furthermore, $(\mathcal{V}(X), \mathscr{G}(X), \bar{\kappa})$ is a locally trivial analytic affine-Banach bundle over $\mathscr{G}(X)$.

Another disguised form of $\mathcal{J}(X)$ is the subspace

$$
\mathscr{G}(X) \times_{\mathfrak{e}} \mathscr{G}(X):=\{(E, F) \in \mathscr{G}(X) \times \mathscr{G}(X): F \top E\}
$$

of $\mathscr{G}(X) \times \mathscr{G}(X)$. We will say some words about this subspace in the following.

Corollary 3.6. Suppose that $\mathcal{T}$ is the topology on $\mathscr{G}(X) \times_{\mathrm{e}} \mathscr{G}(X)$ induced from the product topology on $\mathscr{G}(X) \times \mathscr{G}(X)$. There is a $\mathbb{K}$-Banach manifold structure on $\mathscr{G}(X) \times{ }_{\mathfrak{e}} \mathscr{G}(X)$ compatible with $\mathcal{T}$ such that under the projection $\kappa_{1}: \mathscr{G}(X) \times_{\mathfrak{e}} \mathscr{G}(X) \rightarrow \mathscr{G}(X)$ onto the first coordinate, one obtain a locally trivial analytic affine-Banach bundle structure on $\mathscr{G}(X) \times_{\mathfrak{e}} \mathscr{G}(X)$.

Proof. By Theorem 3.4, it suffices to show that $\Psi:(E, F) \mapsto Q_{E}^{F}$ is a homeomorphism from $\mathscr{G}(X) \times{ }_{\mathfrak{e}} \mathscr{G}(X)$ onto $\mathcal{J}(X)$. Moreover, thanks to the continuity of $\kappa$, it suffices to establish the continuity of $\Psi$. For this, let us consider a sequence $\left\{\left(E_{n}, F_{n}\right)\right\}_{n \in \mathbb{N}}$ in $\mathscr{G}(X) \times_{\mathcal{e}} \mathscr{G}(X)$ converging to $\left(E_{0}, F_{0}\right) \in \mathscr{G}(X) \times{ }_{\mathcal{e}} \mathscr{G}(X)$. By Corollary 3.2, we may assume that $E_{n} \in \mathcal{C}_{F_{0}}$ and $F_{n} \in \mathcal{C}_{E_{0}}$ for all $n \in$ $\mathbb{N}$. Relation (3.13) and Corollary 3.2 produce $Q_{E_{0}}^{\Xi_{E_{0}, F_{0}}\left(E_{n}\right)^{-1}\left(F_{n}\right)} \rightarrow Q_{E_{0}}^{F_{0}}$. Moreover, the continuity of $\Theta_{E_{0}, F_{0}}$ gives the required convergence: $Q_{E_{n}}^{F_{n}}=\Theta_{E_{0}, F_{0}}\left(E_{n}, Q_{E_{0}}^{\Xi_{E_{0}, F_{0}}\left(E_{n}\right)^{-1}\left(F_{n}\right)}\right) \rightarrow Q_{E_{0}}^{F_{0}}$. 
The structure of the above affine-Banach bundle will be state explicitly in the following. For $\left(E_{0}, F_{0}\right) \in \mathscr{G}(X) \times_{\mathfrak{e}} \mathscr{G}(X)$, we define $\check{\mu}_{E_{0}, F_{0}}: \kappa_{1}^{-1}\left(\mathcal{C}_{F_{0}}\right) \rightarrow \mathcal{L}^{F_{0}}\left(X, F_{0}\right) \oplus \mathcal{L}^{E_{0}}\left(X, E_{0}\right)$ to be the map

$$
\check{\mu}_{E_{0}, F_{0}}(E, F):=\left(Q_{E}^{F_{0}}-Q_{E_{0}}^{F_{0}}, Q_{E_{0}}^{F_{0}} Q_{E}^{F} Q_{F_{0}}^{E_{0}}\right) \quad\left(E \in \mathcal{C}_{F_{0}} ; F \in \mathcal{C}_{E}\right) .
$$

Then $\check{\mu}_{E_{0}, F_{0}}^{-1}(R, S)=\left(\left(I_{X}+R\right)\left(S+Q_{E_{0}}^{F_{0}}\right)(X), \operatorname{ker}\left(S+Q_{E_{0}}^{F_{0}}\right)\left(I_{X}-R\right)\right)$ and $\left\{\check{\kappa}^{-1}\left(\mathcal{C}_{F}\right), \check{\mu}_{E, F}, \mathcal{L}^{F}(X, F) \oplus\right.$ $\left.\mathcal{L}^{E}(X, E):(E, F) \in \mathscr{G}(X) \times_{\mathfrak{e}} \mathscr{G}(X)\right\}$ is an analytic atlas for $\mathscr{G}(X) \times_{\mathfrak{e}} \mathscr{G}(X)$.

A direct consequence of Corollary 3.6 is the well-known fact that $\mathscr{G}\left(\mathbb{K}^{n}\right) \times_{\mathrm{e}} \mathscr{G}\left(\mathbb{K}^{n}\right)$ is not closed in $\mathscr{G}\left(\mathbb{K}^{n}\right) \times \mathscr{G}\left(\mathbb{K}^{n}\right)$ for any $n \geq 2$. In fact, $\mathcal{J}\left(\mathbb{K}^{n}\right)$ is never norm compact because it contains non-zero affine-Banach subspaces, but $\mathscr{G}\left(\mathbb{K}^{n}\right) \times \mathscr{G}\left(\mathbb{K}^{n}\right)$ is compact.

\section{4. $J(X)$ AS A BANACH BUNDLE}

It is natural to ask if $(\mathcal{J}(X), \mathscr{G}(X), \kappa)$ is actually a Banach bundle, instead of an affine-Banach bundle. The first proposition in this section is that one can regard $(\mathcal{J}(X), \mathscr{G}(X), \kappa)$ as a continuous Banach bundle.

In fact, Proposition 2.3 tells us that it suffices to show the existence of a continuous global cross section for $(\mathcal{J}(X), \mathscr{G}(X), \kappa)$. In order to construct such a global cross section, let us fix an element $F_{E} \in \mathcal{C}_{E}$ for every $E \in \mathscr{G}(X)$. Since $\mathscr{G}(X)$ is metrizable (see e.g., [15, §2.1]), there is a partition of unity $\left\{\psi_{E}\right\}_{E \in \mathscr{G}(X)}$, consisting of continuous functions, dominated by the open covering $\left\{\mathcal{C}_{F_{E}}\right\}_{E \in \mathscr{G}(X)}$ of $\mathscr{G}(X)$. On the other hand, as $\kappa^{-1}\left(\mathcal{C}_{F_{E}}\right)$ is homeomorphic to a trivial affine-Banach bundle, there exists a local cross section on it. Now, a standard "scaled-sum construction" will produce the required continuous global cross section (observe that as $\mathcal{J}(X)_{E}$ is an affine subspace of $\mathcal{L}(X)$, it is closed under convex combinations).

Let us state this clearly as follows.

Proposition 4.1. If $X$ is a $\mathbb{K}$-Banach space, then $(\mathcal{J}(X), \mathscr{G}(X), \kappa)$ is a locally trivial continuous $\mathbb{K}$-Banach bundle, under equivalent $\mathbb{K}$-Banach space structures on all the fibers of $\kappa$.

By Proposition 2.3(b), the only obstruction for $\mathcal{J}(X)$ to be identified with a locally trivial analytic Banach bundle is the existence of an analytic global cross section. However, a complex analytic global cross section does not exist even in the case when $X=\mathbb{C}^{2}$.

Example 4.2. Let $E_{\lambda}$ be as in Example 3.5. Suppose that there is a complex analytic global cross section $\rho: \mathscr{G}\left(\mathbb{C}^{2}\right) \rightarrow \mathcal{J}\left(\mathbb{C}^{2}\right)$. Then one can find a function $\alpha: \mathbb{C} \backslash\{0\} \rightarrow \mathbb{C}$ satisfying

$$
\rho \circ \pi_{E_{0}, E_{\infty}}^{-1}\left(\left[\begin{array}{ll}
0 & 0 \\
\lambda & 0
\end{array}\right]\right)=\left[\begin{array}{cc}
1-\alpha(\lambda) & \alpha(\lambda) / \lambda \\
\lambda(1-\alpha(\lambda)) & \alpha(\lambda)
\end{array}\right] \quad(\lambda \in \mathbb{C} \backslash\{0\}) .
$$

As $\rho \circ \circ \pi_{E_{0}, E_{\infty}}^{-1}$ is complex analytic, we know that $\alpha$ is holomorphic. Moreover, the compactness of $\mathscr{G}\left(\mathbb{C}^{2}\right)$ tells us that the image of $\rho$ is norm-bounded. From this, we deduce that the three functions

$$
\lambda \mapsto \alpha(\lambda), \quad \lambda \mapsto \alpha(\lambda) / \lambda \quad \text { and } \quad \lambda \mapsto \lambda(1-\alpha(\lambda))
$$

are bounded on $\mathbb{C} \backslash\{0\}$. As $\alpha$ is bounded, it has a removable singularity at 0 . Thus, $\alpha$ extends to a bounded entire function on $\mathbb{C}$, which can only be a constant function. On the other hand, since $\lambda \mapsto \alpha(\lambda) / \lambda$ is bounded as well, we know that $\alpha$ is the constant zero function. However, this will contradict with the boundedness of $\lambda \mapsto \lambda(1-\alpha(\lambda))$. Consequently, there does not exist a complex analytic global cross section on $\mathcal{J}\left(\mathbb{C}^{2}\right)$. In other words, $\left(\mathcal{J}\left(\mathbb{C}^{2}\right), \mathscr{G}\left(\mathbb{C}^{2}\right), \kappa\right)$ is not a locally trivial complex analytic Banach bundle.

More generally, there is no complex differentiable global cross section on $(\mathcal{J}(X), \mathscr{G}(X), \kappa)$ for any complex Banach space $X$. 
Proposition 4.3. Let $X$ be a complex Banach space. We denote by $\mathscr{G}(X)_{\text {fin }}$ the subset of $\mathscr{G}(X)$ consisting of finite dimensional subspaces, and set

$$
\mathcal{J}(X)_{\text {fin }}:=\kappa^{-1}\left(\mathscr{G}(X)_{\text {fin }}\right) .
$$

Then the subbundle $\left(\mathcal{J}(X)_{\mathrm{fin}}, \mathscr{G}(X)_{\mathrm{fin}}, \kappa\right)$ of $(\mathcal{J}(X), \mathscr{G}(X), \kappa)$ is not a locally trivial complex differentiable Banach bundle (see Remark 2.4(b)).

Proof. Suppose on the contrary that $\left(\mathcal{J}(X)_{\text {fin }}, \mathscr{G}(X)_{\text {fin }}, \kappa\right)$ is a locally trivial complex differentiable Banach bundle. Then there is a complex differentiable global cross section $\rho: \mathscr{G}(X)_{\text {fin }} \rightarrow \mathcal{J}(X)_{\text {fin }}$. Consider $E_{0} \in \mathscr{G}(X)_{\text {fin }}$ and $F_{0} \in \mathcal{C}_{E_{0}}$.

Fix an operator $T \in \mathcal{L}\left(E_{0}, F_{0}\right)$ and put $Y:=E_{0}+T\left(E_{0}\right)$. Then $Y$ is a finite dimensional subspace. For any $\lambda \in \mathbb{C}$, one has

$$
p_{E_{0}, F_{0}}^{-1}(\lambda T)=(I+\lambda T)\left(E_{0}\right) \subseteq Y,
$$

where $p_{E_{0}, F_{0}}$ is as in (2.2). Hence, $\left\{p_{E_{0}, F_{0}}^{-1}(\lambda T): \lambda \in \mathbb{C}\right\} \subseteq \mathscr{G}(Y) \subseteq \mathscr{G}(X)_{\mathrm{fin}}$.

Choose any $x \in X$ and any $f$ in the dual space, $X^{*}$, of $X$. As the inclusion map $\iota: \mathcal{J}(X) \rightarrow \mathcal{L}(X)$ is complex analytic, we know that the function $\chi$ on $\mathbb{C}$ defined by

$$
\chi(\lambda):=f\left(\rho\left(p_{E_{0}, F_{0}}^{-1}(\lambda T)\right)(x)\right)
$$

is holomorphic (since (2.4) is an analytic atlas). On the other hand, as $\mathscr{G}(Y)$ is compact and $\left.\rho\right|_{\mathscr{G}(Y)}$ is continuous, we know that

$$
\sup _{E \in \mathscr{G}(Y)}\|\rho(E)\|<\infty .
$$

This means that $\chi$ is bounded and hence it should be a constant function.

Since $x$ and $f$ are arbitrarily chosen, we know that $\lambda \mapsto \rho\left(p_{E_{0}, F_{0}}^{-1}(\lambda T)\right)$ is a constant map, for each fixed $T \in \mathcal{L}\left(E_{0}, F_{0}\right)$. Consequently, the map $\rho \circ p_{E_{0}, F_{0}}^{-1}: \mathcal{L}\left(E_{0}, F_{0}\right) \rightarrow \mathcal{J}(X)$ is constant (since all the rays pass through 0 ), but this contradicts with the fact that $\rho$ is a cross section on $\mathcal{C}_{F_{0}}$.

In the following, we consider the case of (real or complex) Hilbert spaces.

Proposition 4.4. If $H$ is a complex Hilbert space, then $(\mathcal{J}(H), \mathscr{G}(H), \kappa)$ is a locally trivial real analytic complex Banach bundle, under equivalent complex Banach space structures on the fibers such that the self-adjoint projection corresponding to the base point of a fiber is the zero element of the vector space structure on that fiber.

Proof. For any $E \in \mathscr{G}(H)$, we denote by $E^{\perp}$ the orthogonal complement of $E$, and set

$$
P_{E}:=Q_{E}^{E^{\perp}} \text {. }
$$

By the argument of Proposition 2.3(b), the conclusion is obtained if one can show that $E \mapsto P_{E}$ is a real analytic map from $\mathscr{G}(H)$ to $\mathfrak{J}(H)$, or equivalently from $\mathscr{G}(H)$ to $\mathcal{L}(H)$ (because $\mathcal{J}(H)$ is a Banach submanifold of $\mathcal{L}(H)$ ). However, this fact was already proved in [1, Proposition 4(4)] (see also [1, Proposition 4(1)]).

Note that $E \mapsto P_{E}$ is never a complex analytic map from $\mathscr{G}(H)$ to $\mathcal{L}(H)$, because of the proof of Proposition 4.3.

When $K$ is a real Hilbert space, one has the stronger conclusion that $(\mathcal{J}(K), \mathscr{G}(K), \kappa)$ can be identified with the tangent bundle of $\mathscr{G}(K)$. In fact, the total space $\mathbf{T}(\mathscr{G}(K))$ of the tangent bundle of $\mathscr{G}(K)$ is the disjoint union $\biguplus_{E \in \mathscr{G}(K)} \mathcal{L}\left(E, E^{\perp}\right)$ of Banach spaces, equipped with an appropriate Banach manifold structure. By Lemma 3.1, for every $E \in \mathscr{G}(K)$ and $T \in \mathcal{L}\left(E, E^{\perp}\right)$, one knows that $T^{*} \bar{P}_{E^{\perp}}+P_{E}$ is in $\mathcal{J}(X)$ (where $T^{*}$ is the adjoint of $T$ ), where $\bar{P}_{E^{\perp}}$ is the orthogonal projection $P_{E^{\perp}}$ regarding as a map from $K$ to $E^{\perp}$.

Theorem 4.5. Let $K$ be a real Hilbert space. The assignment $(E, T) \mapsto T^{*} \bar{P}_{E^{\perp}}+P_{E}$ induces a fiberwise affine bi-analytic bijection from $\mathbf{T}(\mathscr{G}(K))$ onto $\mathcal{J}(K)$, when $\mathfrak{J}(K)$ is equipped with the Banach submanifold structure induced from $\mathcal{L}(K)$. 
Proof. For any $E \in \mathscr{G}(K)$ and $F \in \mathcal{C}_{E}$, we can identify, via the Banach space isomorphism $\Lambda_{F, E}$ as in (3.5),

$$
\mathcal{L}(F, E) \cong \mathcal{L}^{E}(K, E)
$$

in this case, $T \in \mathcal{L}(F, E)$ is identified with $T \bar{Q}_{F}^{E}$ (note that $\bar{Q}_{E^{\perp}}^{E}=\bar{P}_{E^{\perp}}$ ). We may sometime regard $T \in \mathcal{L}(F, E)$ as an operator from $F$ to $K$ as well.

Let us set

$$
\hat{\mathcal{J}}(K):=\biguplus_{E \in \mathscr{G}(K)} \mathcal{L}\left(E^{\perp}, E\right)
$$

and denote by $\hat{\kappa}: \hat{\mathcal{J}}(K) \rightarrow \mathscr{G}(K)$ the map that sends $T \in \mathcal{L}\left(E^{\perp}, E\right)$ to $E$.

Through identification in (4.1), together with the equality

$$
\mathcal{L}^{E}(K, E)=\mathcal{J}(H)_{E}-P_{E} \quad(E \in \mathscr{G}(K)),
$$

one may equate $(\mathcal{J}(K), \mathscr{G}(K), \kappa)$ with $(\hat{\mathcal{J}}(K), \mathscr{G}(K), \hat{\kappa})$, and obtains a Banach manifold structure on $\hat{\mathfrak{J}}(K)$. In this case, the following is an analytic atlas for this structure on $\hat{\mathfrak{J}}(K)$ :

$$
\left\{\hat{\kappa}^{-1}\left(\mathcal{C}_{E_{0}^{\perp}}\right), \hat{\mu}_{E_{0}}, \mathcal{L}^{E_{0}^{\perp}}\left(K, E_{0}^{\perp}\right) \oplus \mathcal{L}^{E_{0}}\left(K, E_{0}\right): E_{0} \in \mathscr{G}(K)\right\}
$$

where

$$
\hat{\mu}_{E_{0}}(T):=\left(Q_{E}^{E_{0}^{\perp}}-P_{E_{0}}, P_{E_{0}}\left(T \circ P_{E^{\perp}}+P_{E}\right) P_{E_{0}^{\perp}}\right) \quad\left(E \in \mathcal{C}_{E_{0}^{\perp}}, T \in \mathcal{L}\left(E^{\perp}, E\right)\right)
$$

(c.f. (3.20)). Moreover, for $R \in \mathcal{L}^{E_{0}^{\perp}}\left(K, E_{0}^{\perp}\right)$ and $S \in \mathcal{L}^{E_{0}}\left(K, E_{0}\right)$, we have, via (3.21),

$$
\hat{\mu}_{E_{0}}^{-1}(R, S)=\left.(I+R)\left(S+P_{E_{0}}\right)(I-R)\right|_{(I+R)\left(E_{0}\right)^{\perp}} .
$$

In order to verify this proposition, it suffices to show that the fiberwise linear map

$$
\Phi: \hat{\mathcal{J}}(K) \rightarrow \mathbf{T}(\mathscr{G}(K))
$$

that sends an operator to its adjoint is bi-analytic.

To do this, let us fix $E_{0} \in \mathscr{G}(K)$. Consider $E_{1} \in \mathcal{C}_{E_{0}^{\perp}}$ and $T \in \mathcal{L}\left(E_{1}, E_{1}^{\perp}\right)$. We define

$$
\gamma(s):=p_{E_{1}, E_{1}^{\perp}}^{-1}(s T) \quad(s \in(-1,1)) .
$$

We also define

$$
\nu_{E_{0}}(T):=\left(\pi_{E_{0}, E_{0}^{\perp}}\left(E_{1}\right), \Psi(T) \bar{P}_{E_{0}}\right),
$$

where $\Psi(T):=\left(p_{E_{0}, E_{0}^{\perp}} \circ \gamma\right)^{\prime}(0)$; i.e. the derivative of $p_{E_{0}, E_{0}^{\perp}} \circ \gamma$ at 0 . As in [10, p.69 \& p.73],

$$
\left(\biguplus_{E \in \mathcal{C}_{E_{0}^{\perp}}} \mathcal{L}\left(E, E^{\perp}\right), \nu_{E_{0}}, \mathcal{L}^{E_{0}^{\perp}}\left(K, E_{0}^{\perp}\right) \times \mathcal{L}^{E_{0}^{\perp}}\left(K, E_{0}^{\perp}\right)\right)
$$

is a local chart of $\mathbf{T}(\mathscr{G}(K))$ around $E_{0}$.

Now, we are required to know the map $p_{E_{0}, E_{0}^{\perp}} \circ p_{E_{1}, E_{1}^{\perp}}^{-1}$, in order to express $\nu_{E_{0}}(T)$. For this, we need to express the two maps $p_{E_{0}, E_{0}^{\perp}} \circ p_{E_{1}, E_{0}^{\perp}}^{-1}$ and $p_{E_{1}, E_{0}^{\perp}} \circ p_{E_{1}, E_{1}^{\perp}}^{-1}$. Let us put $B:=p_{E_{1}, E_{0}^{\perp}}\left(E_{0}\right)$ (i.e. $\left.(I+B)\left(E_{1}\right)=E_{0}\right)$ and $C:=p_{E_{1}^{\perp}, E_{1}}\left(E_{0}^{\perp}\right)$ (i.e. $\left.(I+C)\left(E_{1}^{\perp}\right)=E_{0}^{\perp}\right)$. Denote

$$
D:=I_{E_{1}}+B \text { and } A:=I_{E_{1}^{\perp}}+C \text {, }
$$

where $I_{E_{1}}$ and $I_{E_{1}^{\perp}}$ are the identity maps on $E_{1}$ and $E_{1}^{\perp}$, respectively. Then $D=\left.\left(I+\pi_{E_{1}, E_{0}^{\perp}}\left(E_{0}\right)\right)\right|_{E_{1}}$ is an operator from $E_{1}$ to $E_{0}$ and $A=\left.\left(I+\pi_{E_{1}^{\perp}, E_{1}}\left(E_{0}^{\perp}\right)\right)\right|_{E_{1}^{\perp}}$ is an operator from $E_{1}^{\perp}$ to $E_{0}^{\perp}$. As in the proof of [32, Lemma 3.12], for any $S \in \mathcal{L}\left(E_{1}, E_{1}^{\perp}\right)$, one has

$$
p_{E_{0}, E_{0}^{\perp}} \circ p_{E_{1}, E_{0}^{\perp}}^{-1} \circ p_{E_{1}, E_{0}^{\perp}} \circ p_{E_{1}, E_{1}^{\perp}}^{-1}(S)=\left(A S\left(I_{E_{1}}-C S\right)^{-1}-B\right) D^{-1}
$$

(note that in [32], the notation $a, b, c$ and $d$ were used, instead of $A, B, C$ and $D$ ). Hence, for $s \in(-1,1)$, we have

$$
\left(p_{E_{0}, E_{0}^{\perp}} \circ \gamma\right)^{\prime}(s)=A T\left(I_{E_{1}}-s C T\right)^{-1} D^{-1}+s A T\left(I_{E_{1}}-s C T\right)^{-2} C T D^{-1},
$$

which gives

$$
\Psi(T)=A T D^{-1}=\left(I+C \bar{P}_{E_{1}^{\perp}}\right) T D^{-1} .
$$


Set $R:=\pi_{E_{0}, E_{0}^{\perp}}\left(E_{1}\right)$. It follows from the equality $B \bar{Q}_{E_{1}}^{E_{0}^{\perp}}=\pi_{E_{1}, E_{0}^{\perp}}\left(E_{0}\right)$ as well as Relation (3.6) that $R=-B \bar{Q}_{E_{1}}^{E_{0}^{\perp}}$. This means that

$$
D=\left.(I-R)\right|_{E_{1}},
$$

and Lemma 2.5 gives $D^{-1}=\left.(I+R)\right|_{E_{0}}$. On the other hand, as $R=Q_{E_{1}}^{E_{0}^{\perp}}-P_{E_{0}}$, we have

$$
C \bar{P}_{E_{1}^{\perp}}=\pi_{E_{1}^{\perp}, E_{1}}\left(E_{0}^{\perp}\right)=Q_{E_{0}^{\perp}}^{E_{1}}-P_{E_{1}^{\perp}}=P_{E_{1}}-R-P_{E_{0}} .
$$

From these, we conclude that

$$
\nu_{E_{0}}(T)=\left(\pi_{E_{0}, E_{0}^{\perp}}\left(E_{1}\right),\left(P_{E_{1}}-\pi_{E_{0}, E_{0}^{\perp}}\left(E_{1}\right)+P_{E_{0}^{\perp}}\right) T \bar{P}_{E_{1}}\left(I+\pi_{E_{0}, E_{0}^{\perp}}\left(E_{1}\right)\right) P_{E_{0}}\right) .
$$

Now, the adjoint map $\Phi$ restricts to a fiberwise linear bijection

$$
\Phi_{E_{0}}: \biguplus_{E \in \mathcal{C}_{E_{0}^{\perp}}} \mathcal{L}\left(E^{\perp}, E\right) \rightarrow \biguplus_{E \in \mathcal{C}_{E_{0}^{\perp}}} \mathcal{L}\left(E, E^{\perp}\right) .
$$

Under the corresponding local charts of $\mathcal{J}(K)$ and $\mathbf{T}(\mathscr{G}(K))$ about $\mathcal{C}_{E_{0}^{\perp}}$, the map $\Phi_{E_{0}}$ is transformed into a map that sends $(R, S) \in \mathcal{L}^{E_{0}^{\perp}}\left(K, E_{0}^{\perp}\right) \oplus \mathcal{L}^{E_{0}}\left(K, E_{0}\right)$ to

$$
\left(R,\left(P_{E_{0}^{\perp}}+P_{(I+R)\left(E_{0}\right)}-R\right)\left(I-R^{*}\right)\left(S^{*}+P_{E_{0}}\right)\left(I+R^{*}\right)(I+R) P_{E_{0}}\right)
$$

(see Relations (4.3) and (4.8)). This shows that $\Phi_{E_{0}}$ is real analytic, because $(I+R)\left(E_{0}\right)=$ $\pi_{E_{0}, E_{0}^{\perp}}^{-1}(R)$ and the assignment $E \mapsto P_{E}$ is a real analytic map (note that [1, Proposition $\left.4(4)\right]$ is also valid in the real case using the same argument).

Conversely, consider again $E_{0} \in \mathscr{G}(K)$ and $E_{1} \in \mathcal{C}_{E_{0}^{\perp}}$. Suppose that $R \in \mathcal{L}^{E_{0}^{\perp}}\left(K, E_{0}^{\perp}\right)$ satisfying $E_{1}=\pi_{E_{0}, E_{0}^{\perp}}^{-1}(R)$, and $S \in \mathcal{L}^{E_{0}^{\perp}}\left(K, E_{0}^{\perp}\right)$. Let $A, B, C$ and $D$ be the operators as in the above. If $T \in \mathcal{L}\left(E_{1}, E_{1}^{\perp}\right)$ such that $\Psi(T) \bar{P}_{E_{0}}=S$, then it follows from (4.5) that

$$
T=\left.A^{-1} S\right|_{E_{0}} D \text {. }
$$

Hence, we have $\nu_{E_{0}}^{-1}(R, S)=\left.A^{-1} S\right|_{E_{0}} D$ (see (4.4)).

Since $A=\left.\left(I+\pi_{E_{1}^{\perp}, E_{1}}\left(E_{0}^{\perp}\right)\right)\right|_{E_{1}^{\perp}}$, it follows from Lemma 2.5 that $A^{-1}=\left.\left(I-\pi_{E_{1}^{\perp}, E_{1}}\left(E_{0}^{\perp}\right)\right)\right|_{E_{0}^{\perp}}$. Consequently, Relations (4.6) and (4.7) (notice that $C \bar{P}_{E_{1}^{\perp}}=\pi_{E_{1}^{\perp}, E_{1}}\left(E_{0}^{\perp}\right)$ ) imply

$$
\nu_{E_{0}}^{-1}(R, S)=\left.\left(I+R+P_{E_{0}}-P_{E_{1}}\right) S(I-R)\right|_{E_{1}} .
$$

This, together with Relation (4.2), tells us that $\Phi_{E_{0}}^{-1}$ is transformed (under the corresponding local charts of $\mathcal{J}(K)$ and $\mathbf{T}(\mathscr{G}(K))$ near $\left.\mathcal{C}_{E_{0}^{\perp}}\right)$ into a map sending $(R, S) \in \mathcal{L}^{E_{0}^{\perp}}\left(K, E_{0}^{\perp}\right) \oplus \mathcal{L}^{E_{0}^{\perp}}\left(K, E_{0}^{\perp}\right)$ to

$$
\left(R, P_{E_{0}}\left(P_{E_{1}}\left(I-R^{*}\right) S^{*}\left(I+R^{*}+P_{E_{0}}-P_{E_{1}}\right) P_{E_{1}^{\perp}}+P_{E_{1}}\right) P_{E_{0}^{\perp}}\right) .
$$

Thus, $\Phi_{E_{0}}^{-1}$ is also real analytic. This completes the proof.

We may also identify the tangent bundle of $\mathscr{G}(K)$ with either $\mathcal{V}(K)$ or $\mathscr{G}(K) \times_{\mathrm{e}} \mathscr{G}(K)$ (see the discussion following Theorem 3.4). On the other hand, one can identify $\mathscr{G}(K)$ with the real Banach submanifold $\mathcal{V}_{\mathrm{sa}}:=\left\{V \in \mathcal{V}(K): V^{*}=V\right\} \backslash\{I,-I\}$.

Note that the corresponding statement of Theorem 4.5 for general Banach spaces is in general false because $\mathcal{L}(E, F)$ may not be isomorphic to $\mathcal{L}(F, E)$ for $E, F \in \mathscr{G}(X)$ with $E \top F$.

In the following, we will consider the case when $H$ is a $\mathbb{K}$-Hilbert space. Let us define a map $\tau: \mathcal{J}(H) \rightarrow \mathscr{G}(H) \times \mathcal{L}(H)$ by

$$
\tau(Q):=(\kappa(Q), \iota(Q)) \quad(Q \in \mathcal{J}(H)),
$$

where $\iota: \mathcal{J}(H) \rightarrow \mathcal{L}(H)$ is the inclusion map. Clearly, $\tau$ is a homeomorphism onto its image. Moreover, as in the proof of Theorem 3.4, $\tau$ is an analytic immersion.

We set

$$
\tilde{\mathcal{J}}(H):=\biguplus_{E \in \mathscr{G}(H)} \mathcal{L}^{E}(H, E),
$$


and define the bundle map $\tilde{\kappa}: \tilde{\mathcal{J}}(H) \rightarrow \mathscr{G}(H)$ canonically. One can see from the proofs of Proposition 4.4 and Theorem 4.5 that $(\tilde{\mathcal{J}}(H), \mathscr{G}(H), \tilde{\kappa})$ is a locally trivial real analytic Banach bundle.

Consider a " $\mathcal{L}(H)$-valued metric" on $\tilde{\mathcal{J}}(H)$ given by

$$
\langle S, T\rangle_{\mathcal{L}(H)}:=S T^{*}, \quad \text { for any } S, T \in \tilde{\mathcal{J}}(H) \text { with } \tilde{\kappa}(S)=\tilde{\kappa}(T) .
$$

Notice that $\langle\cdot, \cdot\rangle_{\mathcal{L}(H)}$ satisfies all the requirements of a metric (i.e. fiberwise inner product) except that it takes values in $\mathcal{L}(H)$ instead of the scalar field. Note also that

$$
\langle S, T\rangle_{\mathcal{L}(H)} \in P_{\tilde{\kappa}(S)} \mathcal{L}(H) P_{\tilde{\kappa}(T)} .
$$

For each $x \in H$, we can also define

$$
\langle S, T\rangle_{x}:=\left\langle S T^{*} x, x\right\rangle_{H} .
$$

Then $\left\{\langle\cdot, \cdot\rangle_{x}\right\}_{x \in H}$ is a family of pseudo-metric on $(\tilde{\mathcal{J}}(H), \mathscr{G}(X), \tilde{\kappa})$ which is separating; in the sense that $S=0$ whenever we have $\langle S, S\rangle_{x}=0$ for all $x \in H$. It is easy to see, via the fiberwise linear analytic immersion induced by $\tau$, that $\langle\cdot, \cdot\rangle_{x}$ is real analytic. On the other hand, if $H$ is separable and $\left\{x_{n}\right\}_{n \in \mathbb{N}}$ is a countable dense subset of $H$, then the sequence of pseudo-metric $\left\{\langle\cdot, \cdot\rangle_{x_{n}}\right\}_{n \in \mathbb{N}}$ is also separating.

Let $\mathscr{G}(H)_{\text {fin }}$ be the subset of $\mathscr{G}(H)$ consisting of finite dimensional subspaces, and we put

$$
\tilde{\mathfrak{T}}(H)_{\text {fin }}:=\tilde{\kappa}^{-1}\left(\mathscr{G}(H)_{\text {fin }}\right) .
$$

Then $\left(\tilde{\mathfrak{J}}(H)_{\text {fin }}, \mathscr{G}(H)_{\text {fin }}, \tilde{\kappa}\right)$ is a locally trivial real analytic Banach bundle. For any $S, T \in \tilde{\mathcal{J}}(H)_{\text {fin }}$ with $\tilde{\kappa}(S)=\tilde{\kappa}(T)$, the operator $\langle S, T\rangle_{\mathcal{L}(H)}$ is of finite rank (see (4.10)). Therefore, we can define a metric $\langle\cdot, \cdot\rangle_{\text {fin }}$ on $\tilde{\mathcal{J}}(H)_{\text {fin }}$ by

$$
\langle S, T\rangle_{\text {fin }}:=\operatorname{Tr}\left(S T^{*}\right), \quad \text { for any } S, T \in \tilde{\mathcal{J}}(H)_{\text {fin }} \text { with } \tilde{\kappa}(S)=\tilde{\kappa}(T),
$$

where $\operatorname{Tr}$ is the canonical densely defined trace on $\mathcal{L}(H)$.

In the particular case when $K$ is a real Hilbert space, we see from the identification as in (4.1) that $\tilde{\mathcal{J}}(K)$ is the same as $\hat{\mathfrak{J}}(K)$. Hence, the above discussion, together with Theorem 4.5, gives the following (notice that both $(F, S) \mapsto\left(F, S+P_{F}\right)$ and $(F, R) \mapsto\left(F, R^{*}\right)$ are real bi-analytic maps from $\mathscr{G}(K) \times \mathcal{L}(K)$ to itself $)$.

Corollary 4.6. Let $K$ be a real Hilbert space.

(a) The assignment $(E, T) \mapsto\left(E, T \bar{P}_{E}\right)$ is analytic immersion from $\mathbf{T}(\mathscr{G}(K))$ to the trivial Banach bundle $\left(\mathscr{G}(K) \times \mathcal{L}(K), \mathscr{G}(K), \kappa_{0}\right)$ which is fiberwise linear. This immersion is a homeomorphism from $\mathbf{T}(\mathscr{G}(K))$ onto the Banach subbundle $\left\{(E, S): E \in \mathscr{G}(K) ; S \in \mathcal{L}^{E^{\perp}}\left(K, E^{\perp}\right)\right\}$.

(b) The $\mathcal{L}(K)$-valued metric $\langle\cdot, \cdot\rangle_{\mathcal{L}(K)}$ as in (4.9) induces a separating family of real analytic pseudometrics on $\mathbf{T}(\mathscr{G}(K))$. If $K$ is separable, then one can find a countable separating family of real analytic pseudo-metrics on $\mathbf{T}(\mathscr{G}(K))$.

(c) The metric $\langle\cdot, \cdot\rangle_{\text {fin }}$ as in (4.11) is a real analytic metric on $\mathbf{T}\left(\mathscr{G}(K)_{\mathrm{fin}}\right)$.

Note that since all the tangent spaces of $\mathscr{G}(K)_{\text {fin }}$ are isomorphic to Hilbert spaces, it is already known that a real analytic metric exists on $\mathbf{T}\left(\mathscr{G}(K)_{\text {fin }}\right)$. The above gives an explicit construction of such a metric.

Notice also that the fiber of the Banach bundle $\hat{\mathcal{J}}(K)$ over $E \in \mathscr{G}(K)$ is a Hilbert $\mathcal{L}\left(E^{\perp}\right)$-module with inner product $\langle S, T\rangle_{0}:=S^{*} T$ (in the usual convention, a Hilbert $C^{*}$-module is a right module and the operator-valued inner product is conjugate linear in the first variable). One may use it to define another family of pseudo-metrics on $\mathbf{T}(\mathscr{G}(K))$. 


\section{5. $\mathcal{J}(X)$ AS A DISJOINT UNION OF HOMOGENEOUS SPACES}

In the case when $X$ is finite dimensional, it is well-known that $\mathscr{G}(X)$ can be identified with a disjoint union of quotients of $G \mathcal{L}(X)$ by closed Lie subgroups. The corresponding fact for $\mathcal{J}(X)$ may also be known. We are going to look at the infinite dimensional situation.

In the following, we will consider the analytic actions Ad and $\alpha$ of $G \mathcal{L}(X)$ on $\mathcal{J}(X)$ and $\mathscr{G}(X)$, respectively (see (3.8) and (3.9)). We have already constructed in the proof of Theorem 3.4 an analytic local right inverse $\Xi_{E_{0}, F_{0}}$ (see (3.14)) for the evaluation map at $E_{0}$ from $G \mathcal{L}(X)$ to the orbit $\alpha\left(G \mathcal{L}(X), E_{0}\right)$, for every $E_{0} \in \mathscr{G}(X)$. The following lemma gives an analytic local right inverse for $\mathrm{Ad}$.

Lemma 5.1. For $\left(E_{0}, F_{0}\right) \in \mathscr{G}(X) \times_{\mathfrak{e}} \mathscr{G}(X)$, the map from $\kappa^{-1}\left(\mathcal{C}_{F_{0}}\right)$ to $G \mathcal{L}(X)$ defined by

$$
\check{\Xi}_{E_{0}, F_{0}}\left(Q_{E}^{F}\right):=\left(Q_{F}^{E}+Q_{E}^{F_{0}}\right)\left(Q_{E}^{F_{0}}+Q_{F_{0}}^{E_{0}}\right) \quad\left(Q_{E}^{F} \in \kappa^{-1}\left(\mathcal{C}_{F_{0}}\right)\right)
$$

is an analytic local right inverse for the evaluation map at $Q_{E_{0}}^{F_{0}}$ from $G \mathcal{L}(X)$ onto the orbit of $Q_{E_{0}}^{F_{0}}$ under the action Ad.

Proof. Consider $Q_{E}^{F} \in \kappa^{-1}\left(\mathcal{C}_{F_{0}}\right)$. We know that both $Q_{E}^{F_{0}}+Q_{F_{0}}^{E_{0}}$ and $Q_{F}^{E}+Q_{E}^{F_{0}}$ are invertible (see Lemmas 2.5 and 3.1). Moreover, one easily check that

$$
\check{\Xi}_{E_{0}, F_{0}}\left(Q_{E}^{F}\right)\left(E_{0}\right)=E \text { and } \check{\Xi}_{E_{0}, F_{0}}\left(Q_{E}^{F}\right)\left(F_{0}\right)=F \text {. }
$$

This implies that $\check{\Xi}_{E_{0}, F_{0}}: \kappa^{-1}\left(\mathcal{C}_{F_{0}}\right) \rightarrow G \mathcal{L}(X)$ is a local right inverse for the evaluation map.

Suppose that $(R, S)=\mu_{E_{0}, F_{0}}\left(Q_{E}^{F}\right)$. It follows from (3.20) and (3.21) that

$$
Q_{E}^{F}=(I+R)\left(S+Q_{E_{0}}^{F_{0}}\right)(I-R)
$$

and $R=Q_{E}^{F_{0}}+Q_{F_{0}}^{E_{0}}-I$. Consequently,

$$
\check{\Xi}_{E_{0}, F_{0}}\left(\mu_{E_{0}, F_{0}}^{-1}(R, S)\right)=\left(I-(I+R)\left(S+Q_{E_{0}}^{F_{0}}\right)(I-R)+R+Q_{E_{0}}^{F_{0}}\right)(R+I),
$$

which is analytic as required.

The existences of local analytic right inverses for the evaluation maps from $G \mathcal{L}(X)$ to the orbits of $\alpha$ and Ad, respectively, imply that these two actions are locally transitive in the sense of [32, Definition 8.20]. For any $Q \in \mathcal{J}(X)$ as well as $E \in \mathscr{G}(X)$, let us set

$$
G \mathcal{L}(X)^{Q}:=\left\{W \in G \mathcal{L}(X): W Q W^{-1}=Q\right\} \quad \text { and } \quad G \mathcal{L}(X)^{E}:=\{W \in G \mathcal{L}(X): W E=E\} .
$$

[32, Proposition 8.21] produces the following result.

Proposition 5.2. Let $X$ be a $\mathbb{K}$-Banach space. Suppose that $Q \in \mathcal{J}(X)$ and $E \in \mathscr{G}(X)$. Then both $G \mathcal{L}(X)^{Q}$ and $G \mathcal{L}(X)^{E}$ are analytic $\mathbb{K}$-Banach Lie subgroups of $G \mathcal{L}(X)$. The orbits $\operatorname{Ad}(G \mathcal{L}(X), Q)$ and $\alpha(G \mathcal{L}(X), E)$ are clopen in $\mathcal{J}(X)$ and $\mathscr{G}(X)$, respectively. Moreover, the canonical bijections from, respectively, $G \mathcal{L}(X) / G \mathcal{L}(X)^{Q}$ and $G \mathcal{L}(X) / G \mathcal{L}(X)^{E}$ onto $\operatorname{Ad}(G \mathcal{L}(X), Q)$ and $\alpha(G \mathcal{L}(X), E)$ are $\mathbb{K}$-bi-analytic.

For any $W \in G \mathcal{L}(X)$, we denote by $[W]_{Q}$ and $[W]_{E}$ the images of $W$ in $G \mathcal{L}(X) / G \mathcal{L}(X)^{Q}$ and $G \mathcal{L}(X) / G \mathcal{L}(X)^{E}$, respectively.

Corollary 5.3. (a) The $\mathbb{K}$-Banach submanifold $\mathcal{J}(X)$ of $\mathcal{L}(X)$ can be identified with a disjoint union of homogeneous spaces of the form $G \mathcal{L}(X) / G \mathcal{L}(X)^{Q}$ for some $Q \in \mathcal{J}(X)$, via the map $\Sigma_{Q}$ : $G \mathcal{L}(X) / G \mathcal{L}(X)^{Q} \rightarrow \mathcal{J}(X)$ given by $\Sigma_{Q}\left([W]_{Q}\right):=W Q W^{-1}$.

(b) The $\mathbb{K}$-Banach manifold $\mathscr{G}(X)$ can be identified with a disjoint union of homogeneous spaces of the form $G \mathcal{L}(X) / G \mathcal{L}(X)^{E}$ for some $E \in \mathscr{G}(X)$, via the map $\Sigma^{E}: G \mathcal{L}(X) / G \mathcal{L}(X)^{E} \rightarrow \mathscr{G}(X)$ given by $\Sigma^{E}\left([W]_{E}\right):=W E$.

(c) Let $Q \in \mathcal{J}(X)$. The assignment $v_{0}:[W]_{Q} \mapsto[W]_{Q(X)}$ is a well-defined $\mathbb{K}$-analytic map from $G \mathcal{L}(X) / G \mathcal{L}(X)^{Q}$ to $G \mathcal{L}(X) / G \mathcal{L}(X)^{Q(X)}$ such that $\kappa \circ \Sigma_{Q}=\Sigma^{Q(X)} \circ v_{0}$. 
Our next question concerns with connected components of $\mathcal{J}(X)$. Note that when $G \mathcal{L}(X)$ is connected, Proposition 5.2 tells us that subsets of the form $\operatorname{Ad}(G \mathcal{L}(X), Q)$ and $\alpha(G \mathcal{L}(X), E)$ are all the components of $\mathcal{J}(X)$ and $\mathscr{G}(X)$, respectively. In this case, if we define an equivalence relation $\sim$ on $\mathscr{G}(X) \times_{\mathfrak{e}} \mathscr{G}(X)$ such that $\left(E_{1}, F_{1}\right) \sim\left(E_{2}, F_{2}\right)$ if and only if $E_{1}$ and $F_{1}$ are Banach space isomorphic to $E_{2}$ and $F_{2}$, respectively, then all the disjoint components of $\mathcal{J}(X)$ are of the form

$$
\left\{Q_{E}^{F}:(E, F) \in \mathscr{G}(X) \times_{e} \mathscr{G}(X) \text { with }(E, F) \sim\left(E_{0}, F_{0}\right)\right\}
$$

for some $\left(E_{0}, F_{0}\right) \in \mathscr{G}(X) \times_{\mathfrak{e}} \mathscr{G}(X)$.

In the case when $H$ is a Hilbert space, Kuiper's theorem tells us that $G \mathcal{L}(H)$ is connected (see [26]; see also [21] for the case when the Hilbert space is non-separable). Therefore, one can determine connected components of both $\mathcal{J}(H)$ and $\mathscr{G}(H)$ through the dimensions of subspaces and those of their orthogonal complements.

However, $G \mathcal{L}(X)$ is in general not connected. Nonetheless, the above still holds for finite dimensional subspaces. We will establish this fact in our next result.

Proposition 5.4. Suppose that $X$ is an infinite dimensional $\mathbb{K}$-Banach space and $n \in \mathbb{N}$. Then $\mathcal{J}_{n}(X):=\{Q \in \mathcal{J}(X): \operatorname{dim} Q(X)=n\}$ and $\mathscr{G}_{n}(X):=\{E \in \mathscr{G}(X): \operatorname{dim} E=n\}$ are connected component of $\mathcal{J}(X)$ and $\mathscr{G}(X)$, respectively.

Proof. In the following, for any $F, F_{1}, F_{2} \in \mathscr{G}(X)$, we will write $F=F_{1} \oplus F_{2}$ if $F_{1} \cap F_{2}=(0)$ and $F=F_{1}+F_{2}$.

By Proposition 5.2, it suffices to show that $\mathcal{J}_{n}(X)$ and $\mathscr{G}_{n}(X)$ are connected. Moreover, since $\kappa$ is a continuous surjection from $\mathcal{J}_{n}(X)$ onto $\mathscr{G}_{n}(X)$, we only need to establish the path connectedness of $\mathcal{J}_{n}(X)$. Fix $E_{1}, E_{2} \in \mathscr{G}_{n}(X)$ as well as $F_{1} \in \mathcal{C}_{E_{1}}$ and $F_{2} \in \mathcal{C}_{E_{2}}$. Notice that $E_{1}+E_{2}$ is finite dimensional, and we fixed a subspace $F \in \mathcal{C}_{E_{1}+E_{2}}$. There exist finite dimensional subspaces $\tilde{E}_{1}$ and $\tilde{E}_{2}$ such that

$$
E_{1} \oplus \tilde{E}_{2}=E_{1}+E_{2}=\tilde{E}_{1} \oplus E_{2} .
$$

We know from Lemma 3.1 that $Q_{E_{1}}^{F_{1}}$ is joined to $Q_{1}:=Q_{E_{1}}^{F \oplus \tilde{E}_{2}}$ by a continuous path in $\mathcal{J}(X)_{E_{1}}$. Similarly, $Q_{E_{2}}^{F_{2}}$ is joined by a continuous path to $Q_{2}:=Q_{E_{2}}^{F \oplus \tilde{E}_{1}}$. Therefore, in order to show that $Q_{E_{1}}^{F_{1}}$ and $Q_{E_{2}}^{F_{2}}$ are in the same connected component, we only need to construct a continuous path joining $Q_{1}$ and $Q_{2}$.

Pick a subspace $E_{3}$ of $F$ with dimension $n$ and choose $\check{F} \in \mathscr{G}(F)$ with $F=E_{3} \oplus \check{F}$. Set $Q_{0}:=Q_{E_{3}}^{\check{F} \oplus\left(E_{1}+E_{2}\right)}$. Under the decomposition $X=E_{3} \oplus\left(\check{F} \oplus \tilde{E}_{2}\right) \oplus E_{1}$, one can write

$$
Q_{0}=\left[\begin{array}{ccc}
I_{E_{3}} & 0 & 0 \\
0 & 0 & 0 \\
0 & 0 & 0
\end{array}\right] \quad \text { and } \quad Q_{1}=\left[\begin{array}{ccc}
0 & 0 & 0 \\
0 & 0 & 0 \\
0 & 0 & I_{E_{1}}
\end{array}\right]
$$

Since $\operatorname{dim} E_{1}=\operatorname{dim} E_{3}=n$, there is an isomorphism $\Phi: E_{1} \rightarrow E_{3}$. For $t \in[0, \pi / 2]$, we set

$$
W_{t}=\left[\begin{array}{ccc}
\cos t \cdot I_{E_{3}} & 0 & \sin t \cdot \Phi \\
0 & I_{\check{F} \oplus \tilde{E}_{2}} & 0 \\
-\sin t \cdot \Phi^{-1} & 0 & \cos t \cdot I_{E_{1}}
\end{array}\right] \in \mathcal{L}(X) .
$$

It is not hard to check that $W_{t} \in G \mathcal{L}(X)$. Moreover, as $W_{0} Q_{0} W_{0}^{-1}=Q_{0}$ and $W_{\pi / 2} Q_{0} W_{\pi / 2}^{-1}=Q_{1}$, we see that $Q_{0}$ and $Q_{1}$ are joined by a continuous path of idempotents.

In the same way, $Q_{0}$ and $Q_{2}$ are joined by a continuous path of idempotents, via the presentations of $Q_{0}$ and $Q_{2}$ under the decomposition $X=E_{3} \oplus\left(\check{F} \oplus \tilde{E}_{1}\right) \oplus E_{2}$. This completes the proof of the connectedness of $\mathcal{J}_{n}(X)$.

In the remainder of this section, we will again look at the case of a $\mathbb{K}$-Hilbert space $H$. For any $Q \in \mathcal{J}(H)$, one can find $P \in \mathcal{J}(H)$ and $W \in G \mathcal{L}(H)$ such that $P^{*}=P$ and $Q=W P W^{-1}$ (see e.g. $[4$, Proposition 4.6.2]). Hence, $\operatorname{Ad}(G \mathcal{L}(H), P)=\operatorname{Ad}(G \mathcal{L}(H), Q)$. This means that in order to study components of $\mathcal{J}(H)$ it suffices to consider component generated by self-adjoint projections. 
Let us denote $\mathcal{U}(H):=\left\{V \in \mathcal{L}(H): V V^{*}=I=V^{*} V\right\}$, and

$$
\mathcal{U}(H)^{P}:=\mathcal{U}(H) \cap G \mathcal{L}(H)^{P} .
$$

It is well-known that $\mathcal{U}(H)$ is a real analytic Banach manifold, and the assignment $V \mapsto V P(H)$ induces a real bi-analytic map from $\mathcal{U}(H) / \mathcal{U}(H)^{P}$ onto a clopen subset of $\mathscr{G}(H)$ (see e.g. [1, Proposition $3(3)])$.

Assume that $k:=\operatorname{dim}_{\mathbb{K}} P(H)<\infty$. Then the image of $\mathcal{U}(H) / \mathcal{U}(H)^{P}$ in $\mathscr{G}(H)$ is precisely the subset $\mathscr{G}_{k}(H)$ of $k$-dimensional subspaces. Moreover, Corollary 5.3(b) and Proposition 5.4 gives

$$
G \mathcal{L}(H) / G \mathcal{L}(H)^{P(H)} \approx \mathscr{G}_{k}(H) .
$$

By Proposition 4.4 as well as Corollary 5.3(c), one obtains a locally trivial real analytic $\mathbb{K}$-Banach bundle $\left(G \mathcal{L}(H) / G \mathcal{L}(H)^{P}, \mathfrak{U}(H) / \mathcal{U}(H)^{P}, v\right)$.

It is natural to ask if there is an explicit way to express this map $v$ (which is defined through several identifications). In the following, we will describe it via the Gram-Schmidt process. Consider $W \in G \mathcal{L}(H)$. We are required to find $V \in \mathcal{U}(H)$ satisfying

$$
W P(H)=V P(H) .
$$

Pick an orthogonal normal basis $\left\{\xi_{1}, \ldots, \xi_{k}\right\}$ for the Hilbert space $P(H)$, and extend it to an orthogonal basis $B$ of $H$. By applying the Gram-Schmidt process to $\left\{W \xi_{1}, \ldots, W \xi_{k}\right\}$, one obtains a collection $\left\{\zeta_{1}, \ldots, \zeta_{k}\right\}$ of orthogonal unit vectors, and we extends it to an orthogonal basis $D$ of $H$. Now, consider $V \in \mathcal{U}(H)$ satisfying $V(B)=D$ and $V \xi_{i}=\zeta_{i}$ for $i \in\{1, \ldots, k\}$. Then one clearly has $W P(H)=V P(H)$. This means that $v\left([W]_{P}\right)=[V]_{P}$.

In the case when $\operatorname{dim} H<\infty$, we can actually applies the Gram-Schmidt process to the finite subset $\{W \xi: \xi \in B\}$ and obtain an element $V \in \mathcal{U}(H)$ satisfying our requirement. In particular, this gives the following.

Example 5.5. (a) Consider an integer $n \geq 2$. Suppose that $Q \in \mathcal{J}\left(\mathbb{K}^{n}\right)$ with $k:=\operatorname{dim}_{\mathbb{K}} Q\left(\mathbb{K}^{n}\right)$. There exists $W \in G \mathcal{L}\left(\mathbb{K}^{n}\right)$ such that $W Q W^{-1}$ is the diagonal matrix $P_{k}$ with first $k$ entries in the diagonal being 1 and all other entries being 0 . In this case, $G \mathcal{L}\left(\mathbb{K}^{n}\right)^{P_{k}}=G \mathcal{L}\left(\mathbb{K}^{k}\right) \times G \mathcal{L}\left(\mathbb{K}^{n-k}\right)$ and $\mathcal{U}\left(\mathbb{K}^{n}\right)^{P_{k}}=\mathcal{U}\left(\mathbb{K}^{k}\right) \times \mathcal{U}\left(\mathbb{K}^{n-k}\right)$. As in the above, the map $v$ from $G \mathcal{L}\left(\mathbb{K}^{n}\right)$ to $\mathcal{U}\left(\mathbb{K}^{n}\right)$ given by the Gram-Schmidt process on the column vectors of matrices produces a map

$$
v: G \mathcal{L}\left(\mathbb{K}^{n}\right) / G \mathcal{L}\left(\mathbb{K}^{k}\right) \times G \mathcal{L}\left(\mathbb{K}^{n-k}\right) \rightarrow \mathcal{U}\left(\mathbb{K}^{n}\right) / \mathcal{U}\left(\mathbb{K}^{k}\right) \times \mathcal{U}\left(\mathbb{K}^{n-k}\right)
$$

and this induces a locally trivial real analytic $\mathbb{K}$-Banach bundle structure on $G \mathcal{L}\left(\mathbb{K}^{n}\right) / G \mathcal{L}\left(\mathbb{K}^{k}\right) \times$ $G \mathcal{L}\left(\mathbb{K}^{n-k}\right)$.

(b) Let $n \geq 2$ and $k \in\{1, \ldots, n-1\}$. Denote by $\mathrm{GL}_{n}$ and $\mathrm{O}_{n}$ the sets of all $n \times n$ real invertible matrices and orthogonal matrices, respectively. Suppose that

$$
v: \mathrm{GL}_{n} / \mathrm{GL}_{k} \times \mathrm{GL}_{n-k} \rightarrow \mathrm{O}_{n} / \mathrm{O}_{k} \times \mathrm{O}_{n-k}
$$

is the map given by the Gram-Schmidt process on the column vectors of matrices. Then there is a bi-analytic bijection from the tangent bundle of $\mathrm{O}_{n} / \mathrm{O}_{k} \times \mathrm{O}_{n-k}$ onto $\mathrm{GL}_{n} / \mathrm{GL}_{k} \times \mathrm{GL}_{n-k}$.

\section{REFERENCES}

[1] M.C. Abbati and A. Manià, A geometrical setting for geometric phases on complex Grassmann manifolds, J. Geom. Phys. 57 (2007), 777-797.

[2] A. Abbondandolo and P. Majer, Infinite dimensional Grassmannians, J. Operator Theory 61 (2009), 19-62.

[3] D. Beltită and J.E. Galé, On complex infinite-dimensional Grassmann manifolds, Complex Anal. Oper. Theory 3 (2009), 739-758.

[4] B. Blackadar, K-theory for operator algebras, 2nd ed., Math. Sci. Research Inst. publ. 5, Cambridge Univ. Press (1998).

[5] F. Botelho, J. Jamison and L. Molnr, Surjective isometries on Grassmann spaces, J. Funct. Anal. 265 (2013), 22262238 .

[6] A.S. Buch, A. Kresch and H. Tamvakis, A Giambelli formula for even orthogonal Grassmannians, J. Reine Angew. Math. 708 (2015), 1748.

[7] L. Costa and R.M. Mir-Roig, $G L(V)$-invariant Ulrich bundles on Grassmannians, Math. Ann. 361 (2015), 443457. 
[8] G. Corach, H. Porta and L. Recht, The geometry of spaces of projections in $C^{*}$-algebras, Adv. Math. 101 (1993), 59-77.

[9] C.H. Chu, Grassmann manifolds of Jordan algebras, Arch. Math. (Basel) 87 (2006), 179-192.

[10] C.H. Chu, Jordan structures in geometry and analysis, Camb. Tracts in Math. 190, Camb. Univ. Press (2012).

[11] C.H. Chu and J.M. Isidro, Manifolds of tripotents in $J B^{*}$-triples, Math. Z. 233 (2000), 741-754.

[12] H. De Sterck and A. Howse, Nonlinearly preconditioned optimization on Grassmann manifolds for computing approximate Tucker tensor decompositions, SIAM J. Sci. Comput. 38 (2016), A997A1018.

[13] R.S. Doran and J.M.G. Fell, Representations of ${ }^{*}$-algebras, locally compact groups, and Banach ${ }^{*}$-algebraic bundles, volume 1, Pure and applied mathematics 125, Academic Press, Boston (1988).

[14] A. Douady, Un espace de Banach dont le groupe linéaire n'est pas connexe, Indag. Math. 27 (1965), 787-789.

[15] A. Douady, Le problème des modules pour les sous-espaces analytiques compacts d'un espace analytique donné, Ann. Inst. Fourier (Grenoble) 16 (1966), no. 1, 1-95.

[16] M.J. Dupré and R.M. Gillette, Banach bundles, Banach Modules and Automorphisms of $C^{*}$-algebras, Pitman Research Notes in Mathematics 92, John Wiley \& Sons, Inc., New York, 1983.

[17] M. Farber and A. Postnikov, Arrangements of equal minors in the positive Grassmannian, Adv. Math. 300 (2016), 788834 .

[18] S. Fiori, T. Kaneko and T. Tanaka, Tangent-bundle maps on the Grassmann manifold: application to empirical arithmetic averaging, IEEE Trans. Signal Process. 63 (2015), 155168.

[19] G. Gehér and Šemrl, Isometries of Grassmann spaces, J. Funct. Anal. 270 (2016), 15851601.

[20] J. Guo, Z. Lu and E. Sharpe, Quantum sheaf cohomology on Grassmannians, Comm. Math. Phys. 352 (2017), 135184 .

[21] Luc Illusie, Contractibilité du groupe linéaire des espaces de Hilbert de dimension infinie, Séminaire Bourbaki, Exp. No. 284, (1964).

[22] A. Ito, On birational geometry of the space of parametrized rational curves in Grassmannians, Trans. Amer. Math. Soc. 369 (2017), 62796301.

[23] W. Kaup, Über die automorphismen grassmannscher mannigfaltigkeiten unendlicher dimension, Math. Z. 144 (1975) 75-96.

[24] W. Kaup, On Grassmannians associated with $J B^{*}$-triples, Math. Z. 236 (2001), 567-584.

[25] Y. Kodama and L. Williams, KP solitons and total positivity for the Grassmannian, Invent. Math. 198 (2014), 637699.

[26] N.H. Kuiper, The homotopy type of the unitary group of a Hilbert space, Topology 3 (1965), 19-30.

[27] S. Lang, Differential and Riemannian Manifolds, GTM 160, Springer-Verlag (1995).

[28] A.J. Li, D. Xi and G. Zhang, Volume inequalities of convex bodies from cosine transforms on Grassmann manifolds, Adv. Math. 304 (2017), 494538.

[29] M. Nokleby, M. Rodrigues and R. Calderbank, Discrimination on the Grassmann manifold: fundamental limits of subspace classifiers, IEEE Trans. Inform. Theory 61 (2015), 21332147.

[30] H. Porta and L. Recht, Spaces of projections in a Banach algebra, Acta Cient. Venezolana 38 (1987), 408-426.

[31] H. Schröder, On the topology of the group of invertible elements - A Survey, preprint (arXiv:Math/9810069v1).

[32] H. Upmeier, Symmetric Banach manifolds and Jordan $C^{*}$-algebras, North-Holland mathematics studies 104, North-Holland, Amsterdam (1985).

[33] X. Zeng, W. Bian, W. Liu, J. Shen and D. Tao, Dictionary pair learning on Grassmann manifolds for image denoising, IEEE Trans. Image Process. 24 (2015), 45564569.

(Chi-Wai Leung) Department of Mathematics, The Chinese University of Hong Kong, Hong Kong.

E-mail address: cwleung@math.cuhk.edu.hk

(Chi-Keung Ng) Chern Institute of Mathematics and LPMC, Nankai University, Tianjin 300071, China.

E-mail address: ckng@nankai.edu.cn 